\title{
On a generalization of Eulerian numbers
}

\author{
Claudio Pita-Ruiz \\ Facultad de Ingeniería, Universidad Panamericana \\ Augusto Rodin 498, México, Ciudad de México, 03920, México \\ e-mail: cpita@up.edu.mx
}

Received: 27 July 2016

Accepted: 31 January 2018

\begin{abstract}
We consider the sequence $\left(\begin{array}{c}a+b \\ r\end{array}\right)^{p} P(n)$, product of the $r p$-th degree $n$-polynomial $\left(\begin{array}{c}a n+b \\ r\end{array}\right)^{p}$, where $a, b \in \mathbb{C}, a \neq 0, r, p \in \mathbb{N}$, and the $\left(\sum_{s=2}^{l} r_{s} p_{s}\right)$-th degree $n$-polynomial $P(n)=$ $\prod_{s=2}^{l}\left(\begin{array}{c}\alpha_{s} n+\beta_{s} \\ r_{s}\end{array}\right)^{p_{s}}$, where $\alpha_{s}, \beta_{s} \in \mathbb{C}, r_{s}, p_{s} \in \mathbb{N}, s=2, \ldots, l$. In the expansion of the polynomial $\left(\begin{array}{c}a n+b \\ r\end{array}\right)^{p} P(n)$ in terms of the binomials $\left(\begin{array}{c}n+r p+\sum_{s=2}^{l} r_{s} p_{s}-i \\ r p+\sum_{s=2}^{l} r_{s} p_{s}\end{array}\right), i=0,1, \ldots, r p+\sum_{s=2}^{l} r_{s} p_{s}$, the resulting coefficients $A_{a, b, r}^{\left(\alpha_{s}, \beta_{s}, r_{s}, p_{s}\right)}(p, i)$ are the generalized Eulerian numbers we consider in this work (the case $P(n)=1, a=1, b=0, r=1$ corresponds to the standard Eulerian numbers). We obtain results on symmetries, recurrences, row sums, and alternating row sums, that generalize the corresponding well-known results for the standard Eulerian numbers. The main tool we use to obtain our results throughout the work, is the $Z$-transform of sequences.
\end{abstract}

Keywords: Generalized Eulerian numbers.

2010 Mathematics Subject Classification: 11B83.

\section{Introduction}

Eulerian numbers have been mathematical (combinatorial) objects of interest to mathematicians along the years: beginning with Euler's work [10] in the eighteenth century, they are still considered as objects worthy to study $[11,12]$. We have nowadays several generalizations of these objects [17, 18, 19, 20, 25]. Within the works of L. Carlitz (besides the expository work [3]), we find also some generalizations of Eulerian numbers [6,7], including $q$-generalizations of them $[2,4,5]$ (among other related works [8]).

Eulerian numbers appear as coefficients of the sequence of polynomials $1, z+1, z^{2}+4 z+1$, $z^{3}+11 z^{2}+11 z+1, \ldots$ (called Eulerian polynomials), considered by L. Euler ([10], pp. 485, 486). 
We will use the notation $A(p, i)$ for the corresponding Eulerian number in the $p$-th row and $i$-th column of the so-called Eulerian numbers triangle, with $p=1,2, \ldots$ (rows), and $i=0,1,2, \ldots$ (columns), namely

\begin{tabular}{c||cccccc}
$p \backslash i$ & 0 & 1 & 2 & 3 & 4 & $\cdots$ \\
\hline \hline 1 & 0 & 1 & & & & \\
2 & 0 & 1 & 1 & & & $\cdots$ \\
3 & 0 & 1 & 4 & 1 & & \\
4 & 0 & 1 & 11 & 11 & 1 & $\cdots$ \\
$\vdots$ & & $\vdots$ & & $\vdots$ & &
\end{tabular}

(Some shifted versions of this triangle appear in the literature also as Eulerian numbers triangle.) Some important known facts about Eulerian numbers are the following:

- Explicit formula:

$$
A(p, i)=\sum_{j=0}^{i}(-1)^{j}\left(\begin{array}{c}
p+1 \\
j
\end{array}\right)(i-j)^{p} .
$$

- Symmetry:

$$
A(p, i)=A(p, p+1-i)
$$

- Recurrence:

$$
A(p, i)=i A(p-1, i)+(p+1-i) A(p-1, i-1) .
$$

- Row sums:

$$
\sum_{i=0}^{p} A(p, i)=p !
$$

- Alternating row sums:

$$
\sum_{i=0}^{p}(-1)^{i} A(p, i)=\frac{2^{p+1}\left(1-2^{p+1}\right) B_{p+1}}{p+1},
$$

where $B_{p+1}$ is the $(p+1)$-th Bernoulli number.

Besides the mentioned properties, a remarkable fact is that Eulerian numbers are the coefficients appearing when we write $n^{p}$ as a linear combination of the binomials $\left(\begin{array}{c}n \\ p\end{array}\right),\left(\begin{array}{c}n+1 \\ p\end{array}\right), \ldots,\left(\begin{array}{c}n+p \\ p\end{array}\right)$. This is the Worpitsky identity [24]:

$$
n^{p}=\sum_{i=0}^{p} A(p, i)\left(\begin{array}{c}
n+p-i \\
p
\end{array}\right) .
$$

We will work with the $Z$-transform of sequences, which is a map $\mathcal{Z}$ that takes complex sequences $a_{n}=\left(a_{0}, a_{1}, \ldots, a_{n}, \ldots\right)$ into complex functions $\mathcal{Z}\left(a_{n}\right)(z)$ (or simply $\mathcal{Z}\left(a_{n}\right)$ ) given by the Laurent series $\mathcal{Z}\left(a_{n}\right)=\sum_{n=0}^{\infty} \frac{a_{n}}{z^{n}}$ (defined for $|z|>R$, where $R>0$ is the radius of convergence of the Taylor series $\sum_{n=0}^{\infty} a_{n} z^{n}$ - the generating function of the sequence $a_{n}-$ ). 
If $\mathcal{Z}\left(a_{n}\right)=A(z)$, we also say that the sequence $a_{n}$ is the inverse $Z$-transform of the complex function $A(z)$, and we write $a_{n}=\mathcal{Z}^{-1}(A(z))$. We will recall now some basic facts about the $Z$ transform, that we will use throughout the work (for further reading, see [14, 23]).

The sequence $\lambda^{n}$ (where $\lambda$ is a given non-zero complex number), has $Z$-transform

$$
\mathcal{Z}\left(\lambda^{n}\right)=\sum_{n=0}^{\infty} \frac{\lambda^{n}}{z^{n}}=\frac{1}{1-\frac{\lambda}{z}}=\frac{z}{z-\lambda},
$$

defined for $|z|>|\lambda|$. In particular, the $Z$-transform of the constant sequence 1 is

$$
\mathcal{Z}(1)=\frac{z}{z-1}
$$

Four important properties of the $Z$-transform (which formal proofs are easy exercises left to the reader), are the following:

1. $\mathcal{Z}$ is linear and injective. (We will be using this fact without further comments.)

2. (Advance-shifting property) If $\mathcal{Z}\left(a_{n}\right)=\mathcal{A}(z)$, and $k \in \mathbb{N}$ is given, then

$$
\mathcal{Z}\left(a_{n+k}\right)=z^{k}\left(\mathcal{A}(z)-\sum_{j=0}^{k-1} \frac{a_{j}}{z^{j}}\right) .
$$

3. (Multiplication by the sequence $n$ ) If $\mathcal{Z}\left(a_{n}\right)=\mathcal{A}(z)$, then

$$
\mathcal{Z}\left(n a_{n}\right)=-z \frac{d}{d z} \mathcal{A}(z)
$$

Formula (10) implies

$$
\mathcal{Z}\left(n^{2} a_{n}\right)=z^{2} \frac{d^{2}}{d z^{2}} \mathcal{A}(z)+z \frac{d}{d z} \mathcal{A}(z)
$$

From (8) and (10), we see that the $Z$-transform of the sequence $n$ is

$$
\mathcal{Z}(n)=-z \frac{d}{d z} \frac{z}{z-1}=\frac{z}{(z-1)^{2}}
$$

Similarly, we have that

$$
\begin{aligned}
\mathcal{Z}\left(n^{2}\right) & =-z \frac{d}{d z} \frac{z}{(z-1)^{2}}=\frac{z(z+1)}{(z-1)^{3}} \\
\mathcal{Z}\left(n^{3}\right) & =-z \frac{d}{d z} \frac{z(z+1)}{(z-1)^{3}}=\frac{z\left(z^{2}+4 z+1\right)}{(z-1)^{4}}
\end{aligned}
$$

and so on.

The $Z$-transform of the sequence $\left(\begin{array}{l}n \\ r\end{array}\right)$, where $r \in \mathbb{N}$ is given, is

$$
\mathcal{Z}\left(\left(\begin{array}{l}
n \\
r
\end{array}\right)\right)=\frac{z}{(z-1)^{r+1}} .
$$


(The proof is an easy induction on $r$, left to the reader.) According to the advance-shifting property (9), together with (15), we see that for $0 \leq k \leq r$, we have

$$
\mathcal{Z}\left(\left(\begin{array}{c}
n+k \\
r
\end{array}\right)\right)=\frac{z^{k+1}}{(z-1)^{r+1}} .
$$

Observe that, according to Worpitsky identity (6) and formula (16), the $Z$-transform of the sequence $n^{p}(p \in \mathbb{N}$ given) is

$$
\mathcal{Z}\left(n^{p}\right)=\frac{\sum_{i=0}^{p} A(p, i) z^{p-i+1}}{(z-1)^{p+1}}
$$

It is a known fact that the $p+1$ binomials $\left(\begin{array}{c}n+p-i \\ p\end{array}\right), i=0,1, \ldots, p$ form a basis of the vector space of polynomials of degree at most $p$ (see [22], Sec. 4.3): Worpitsky identity (6) gives us an explicit formula expressing the $p$-th degree polynomial $n^{p}$ as a linear combination of the elements of the mentioned basis, and Eulerian numbers are the corresponding coordinates. Thus, for any polynomial $f$ with $\operatorname{deg} f \leq p$, we can write

$$
f(n)=\sum_{i=0}^{p} c_{i}\left(\begin{array}{c}
n+p-i \\
p
\end{array}\right)
$$

with uniquely determined constants $c_{i}$ depending only on $f$. As Stanley [22] suggests, a good name for these constants is $f$-Eulerian numbers. For example, Shanks [21] considers the $r p$-th degree polynomial $f(n)=\left(\begin{array}{l}n \\ r\end{array}\right)^{p}$, and shows that $f(n)=\sum_{i=0}^{r p} c_{i}\left(\begin{array}{c}n+r p-i \\ r p\end{array}\right)$ for some constants $c_{i}$ (that "do not depend on $n$ "). He gives an explicit expression for $c_{i}$ in terms of a determinant (obtained from Cramer's rule... not really friendly for algebraic manipulation). First noticed by Carlitz [1] (see also [16]), the constants $c_{i}=A_{f}(p, i)$ in the expansion (18) of the polynomial $f(n)$ in terms of binomials $\left(\begin{array}{c}n+p-i \\ p\end{array}\right), i=0,1, \ldots, p$, are

$$
c_{i}=\sum_{j=0}^{i}(-1)^{j}\left(\begin{array}{c}
p+1 \\
j
\end{array}\right) f(i-j) .
$$

These are then the $f$-Eulerian numbers associated to polynomial $f$ of degree at most $p$. Then, the expansion (18) can be written as

$$
f(n)=\sum_{i=0}^{p} \sum_{j=0}^{i}(-1)^{j}\left(\begin{array}{c}
p+1 \\
j
\end{array}\right) f(i-j)\left(\begin{array}{c}
n+p-i \\
p
\end{array}\right) .
$$

In the present work we consider $f$-Eulerian numbers for some general polynomials $f$ (including the Shanks' ones), and study some of their properties. In particular, we consider polynomials which are powers of binomial coefficients $f(n)=\left(\begin{array}{c}a n+b \\ r\end{array}\right)^{p}$, (which is a polynomial of degree $r p$ ), where $a, b$ are given complex numbers, $a \neq 0$, and $r \in \mathbb{N}$ is also given. More generally, we consider polynomials of the type

$$
f(n)=\left(\begin{array}{c}
a n+b \\
r
\end{array}\right)^{p}\left(\begin{array}{c}
\alpha_{2} n+\beta_{2} \\
r_{2}
\end{array}\right)^{p_{2}} \ldots\left(\begin{array}{c}
\alpha_{l} n+\beta_{l} \\
r_{l}
\end{array}\right)^{p_{l}}
$$


where $\alpha_{s}, \beta_{s} \in \mathbb{C}$, and $r_{s}$ and $p_{s}$ non-negative integers, are given, $s=2, \ldots, l$. In this case $f$ is a $\left(r p+\sum_{s=2}^{l} r_{s} p_{s}\right)$-th degree polynomial. For each $p \in \mathbb{N}$, the polynomial (21) can be written as a linear combination of the $\left(r p+\sum_{s=2}^{l} r_{s} p_{s}\right)+1$ binomials $\left(\begin{array}{c}n+r p+\sum_{s=2}^{l} r_{s} p_{s}-i \\ r p+\sum_{s=2}^{l} r_{s} p_{s}\end{array}\right)$, $i=0,1, \ldots, r p+\sum_{s=2}^{l} r_{s} p_{s}$. To study the coefficients of this linear combination is the main goal of this work. In Section 2 we introduce our generalized Eulerian numbers, give some special values of them, and (in Subsection 2.1) we write explicitly the first few rows of some triangles of these numbers. Sections 3, 4, 5 and 6 are devoted to establish generalizations of the properties of symmetry (2), recurrence (3), row sums (4), and alternating row sums (5), respectively.

Finally we comment that our main interest throughout the work is to have "good generalizations" of the well-known 'algebraic' nature and properties of standard Eulerian numbers. We do not consider the combinatorial part of the life of Eulerian numbers: we think that surely there are interesting combinatorial interpretations for the generalized Eulerian numbers considered in this work. This should give material for the future.

\section{The generalized Eulerian numbers}

Note: We will be writing $\sigma$ for $\sum_{s=2}^{l} r_{s} p_{s}$.

Having in mind the principal $r p$-th degree polynomial $\left(\begin{array}{c}a n+b \\ r\end{array}\right)^{p}$, we will consider the product (21), which is a $(r p+\sigma)$-th degree polynomial, and write the expansion (20)

$$
\left(\begin{array}{c}
a n+b \\
r
\end{array}\right)^{p} \prod_{s=2}^{l}\left(\begin{array}{c}
\alpha_{s} n+\beta_{s} \\
r_{s}
\end{array}\right)^{p_{s}}=\sum_{i=0}^{r p+\sigma} A_{a, b, r}^{\left(a_{s}, b_{s}, r_{s}, p_{s}\right)}(p, i)\left(\begin{array}{c}
n+r p+\sigma-i \\
r p+\sigma
\end{array}\right) .
$$

The coefficients

$$
A_{a, b, r}^{\left(a_{s}, b_{s}, r_{s}, p_{s}\right)}(p, i)=\sum_{j=0}^{i}(-1)^{j}\left(\begin{array}{c}
r p+\sigma+1 \\
j
\end{array}\right)\left(\begin{array}{c}
a(i-j)+b \\
r
\end{array}\right)^{p}\left(\prod_{s=2}^{l}\left(\begin{array}{c}
\alpha_{s}(i-j)+\beta_{s} \\
r_{s}
\end{array}\right)^{p_{s}}\right)
$$

where, $i=0,1, \ldots, r p+\sigma$, are the generalized Eulerian numbers we want to study in this work. The notation $A_{a, b, r}^{\left(a_{s}, b_{s}, r_{s}, p_{s}\right)}(p, i)$ suggests that the principal polynomial is $\left(\begin{array}{c}a n+b \\ r\end{array}\right)$, and that this is accompanied (in a product) by polynomials $\left(\begin{array}{c}\alpha_{s} n+\beta_{s} \\ r_{s}\end{array}\right)^{p_{s}}, s=2, \ldots, l$. We have $A_{a, b, r}^{\left(a_{s}, b_{s}, r_{s}, p_{s}\right)}(p, i)=$ 0 for $i<0$ or $i>r p+\sigma$.

When $p_{2}=\cdots=p_{l}=0$ or $r_{2}=\cdots=r_{l}=0$, we have the expansion of the $r p$-th degree polynomial $\left(\begin{array}{c}a+b \\ r\end{array}\right)^{p}$, namely

$$
\left(\begin{array}{c}
a n+b \\
r
\end{array}\right)^{p}=\sum_{i=0}^{r p} A_{a, b, r}(p, i)\left(\begin{array}{c}
n+r p-i \\
r p
\end{array}\right)
$$

which coefficients are the generalized Eulerian numbers

$$
A_{a, b, r}(p, i)=\sum_{j=0}^{i}(-1)^{j}\left(\begin{array}{c}
r p+1 \\
j
\end{array}\right)\left(\begin{array}{c}
a(i-j)+b \\
r
\end{array}\right)^{p}
$$


and in turn, when in these numbers (25) we have $a=1, b=0, r=1$, the resulting numbers $A_{1,0,1}(p, i)$ are the standard Eulerian numbers (1).

According to (16), (22) and (23), the $Z$-transform of the sequence $\left(\begin{array}{c}a n+b \\ r\end{array}\right)^{p} \prod_{s=2}^{l}\left(\begin{array}{c}\alpha_{s} n+\beta_{s} \\ r_{s}\end{array}\right)^{p_{s}}$ is

$$
\mathcal{Z}\left(\left(\begin{array}{c}
a n+b \\
r
\end{array}\right)^{p} \prod_{s=2}^{l}\left(\begin{array}{c}
\alpha_{s} n+\beta_{s} \\
r_{s}
\end{array}\right)^{p_{s}}\right)=\frac{z \sum_{i=0}^{r p+\sigma} A_{a, b, r}^{\left(a_{s}, b_{s}, r_{s}, p_{s}\right)}(p, i) z^{r p+\sigma-i}}{(z-1)^{r p+\sigma+1}}
$$

In particular we have

$$
\mathcal{Z}\left(\left(\begin{array}{c}
a n+b \\
r
\end{array}\right)^{p}\right)=\frac{z \sum_{i=0}^{r p} A_{a, b, r}(p, i) z^{r p-i}}{(z-1)^{r p+1}} .
$$

The first two elements of the $p$-th row in the generalized Eulerian numbers $A_{a, b, r}^{\left(a_{s}, b_{s}, r_{s}, p_{s}\right)}(p, i)$ triangle are (from (23) with $i=0,1$ )

$$
\begin{aligned}
& A_{a, b, r}^{\left(\alpha_{s}, \beta_{s}, r_{s}, p_{s}\right)}(p, 0)=\left(\begin{array}{c}
b \\
r
\end{array}\right)^{p} \prod_{s=2}^{l}\left(\begin{array}{c}
\beta_{s} \\
r_{s}
\end{array}\right)^{p_{s}} \\
& A_{a, b, r}^{\left(\alpha_{s}, \beta_{s}, r_{s}, p_{s}\right)}(p, 1)=\left(\begin{array}{c}
a+b \\
r
\end{array}\right)^{p} \prod_{s=2}^{l}\left(\begin{array}{c}
\alpha_{s}+\beta_{s} \\
r_{s}
\end{array}\right)^{p_{s}}-(r p+\sigma+1)\left(\begin{array}{l}
b \\
r
\end{array}\right)^{p} \prod_{s=2}^{l}\left(\begin{array}{c}
\beta_{s} \\
r_{s}
\end{array}\right)^{p_{s}} .
\end{aligned}
$$

In particular, if $0 \leq b<r$, we have $A_{a, b, r}^{\left(\alpha_{s}, \beta_{s}, r_{s}, p_{s}\right)}(p, 0)=0$. This happens, for example, when $b=0$ (see Remark after formula (35) and triangle GENT2, Subsection 2.1).

Similarly, the last element of the $p$-th row (corresponding to $i=r p+\sigma$ ) is

$$
\begin{aligned}
& A_{a, b, r}^{\left(\alpha_{s}, \beta_{s}, r_{s}, p_{s}\right)}(p, r p+\sigma) \\
= & \sum_{j=0}^{r p+\sigma}(-1)^{j}\left(\begin{array}{c}
r p+\sigma+1 \\
j
\end{array}\right)\left(\begin{array}{c}
a(r p+\sigma-j)+b \\
r
\end{array}\right)^{p} \prod_{s=2}^{l}\left(\begin{array}{c}
\alpha_{s}(r p+\sigma-j)+\beta_{s} \\
r_{s}
\end{array}\right)^{p_{s}} .
\end{aligned}
$$

We claim that (30) can be written as

$$
A_{a, b, r}^{\left(\alpha_{s}, \beta_{s}, r_{s}, p_{s}\right)}(p, r p+\sigma)=\left(\begin{array}{c}
a-b+r-1 \\
r
\end{array}\right)^{p} \prod_{s=2}^{l}\left(\begin{array}{c}
\alpha_{s}-\beta_{s}+r_{s}-1 \\
r_{s}
\end{array}\right)^{p_{s}} .
$$

In fact, we have

$$
\begin{aligned}
& A_{a, b, r}^{\left(\alpha_{s}, \beta_{s}, r_{s}, p_{s}\right)}(p, r p+\sigma+1) \\
= & \sum_{j=0}^{r p+\sigma+1}(-1)^{j}\left(\begin{array}{c}
r p+\sigma+1 \\
j
\end{array}\right)\left(\begin{array}{c}
a(r p+\sigma+1-j)+b \\
r
\end{array}\right) \prod_{s=2}^{p}\left(\begin{array}{c}
\alpha_{s}(r p+\sigma+1-j)+\beta_{s} \\
r_{s}
\end{array}\right)^{p_{s}} \\
= & (-1)^{r p+\sigma+1}\left(\begin{array}{l}
b \\
r
\end{array}\right)^{p}\left(\prod_{s=2}^{l}\left(\begin{array}{l}
\beta_{s} \\
r_{s}
\end{array}\right)^{p_{s}}\right) \\
& +\sum_{j=0}^{r p+\sigma}(-1)^{j}\left(\begin{array}{c}
r p+\sigma+1 \\
j
\end{array}\right)\left(\begin{array}{c}
a(r p+\sigma-j)+b+a \\
r
\end{array}\right)^{p} \prod_{s=2}^{l}\left(\begin{array}{c}
\alpha_{s}(r p+\sigma-j)+\beta_{s}+\alpha_{s} \\
r_{s}
\end{array}\right)^{p_{s}} \\
= & 0 .
\end{aligned}
$$


That is, from (32) we see that

$$
\begin{aligned}
& \sum_{j=0}^{r p+\sigma}(-1)^{j}\left(\begin{array}{c}
r p+\sigma+1 \\
j
\end{array}\right)\left(\begin{array}{c}
a(r p+\sigma-j)+b+a \\
r
\end{array}\right)^{p} \prod_{s=2}^{l}\left(\begin{array}{c}
\alpha_{s}(r p+\sigma-j)+\beta_{s}+\alpha_{s} \\
r_{s}
\end{array}\right)^{p_{s}} \\
= & (-1)^{r p+\sigma}\left(\begin{array}{l}
b \\
r
\end{array}\right)^{p}\left(\prod_{s=2}^{l}\left(\begin{array}{c}
\beta_{s} \\
r_{s}
\end{array}\right)^{p_{s}}\right) .
\end{aligned}
$$

In (33) replace $b$ by $b-a$ and $\beta_{s}$ by $\beta_{s}-\alpha_{s}$ to get

$$
\begin{aligned}
& \sum_{j=0}^{r p+\sigma}(-1)^{j}\left(\begin{array}{c}
r p+\sigma+1 \\
j
\end{array}\right)\left(\begin{array}{c}
a(r p+\sigma-j)+b \\
r
\end{array}\right)^{p} \prod_{s=2}^{l}\left(\begin{array}{c}
\alpha_{s}(r p+\sigma-j)+\beta_{s} \\
r_{s}
\end{array}\right)^{p_{s}} \\
= & (-1)^{r p+\sigma}\left(\begin{array}{c}
b-a \\
r
\end{array}\right)^{p}\left(\prod_{s=2}^{l}\left(\begin{array}{c}
\beta_{s}-\alpha_{s} \\
r_{s}
\end{array}\right)^{p_{s}}\right) .
\end{aligned}
$$

Thus, according to (30), expression (34) is

$$
A_{a, b, r}^{\left(\alpha_{s}, \beta_{s}, r_{s}, p_{s}\right)}(p, r p+\sigma)=(-1)^{r p+\sigma}\left(\begin{array}{c}
b-a \\
r
\end{array}\right)^{p}\left(\prod_{s=2}^{l}\left(\begin{array}{c}
\beta_{s}-\alpha_{s} \\
r_{s}
\end{array}\right)^{p_{s}}\right),
$$

or

$$
A_{a, b, r}^{\left(\alpha_{s}, \beta_{s}, r_{s}, p_{s}\right)}(p, r p+\sigma)=\left(\begin{array}{c}
a-b+r-1 \\
r
\end{array}\right)^{p} \prod_{s=2}^{l}\left(\begin{array}{c}
\alpha_{s}-\beta_{s}+r_{s}-1 \\
r_{s}
\end{array}\right)^{p_{s}}
$$

which proves our claim (31). In particular, if $1-r \leq a-b<1$, we have $A_{a, b, r}^{\left(\alpha_{s}, \beta_{s}, r_{s}, p_{s}\right)}(p, r p+\sigma)=$ 0 (this happens, for example, when $a=b$ ).

Let us consider (as an example) the generalized Eulerian numbers $A_{3,-5,4}^{(1,7,2,1)}(p, i)$ : the first two elements (according to (28) and (29)), and the last element (according to (31)), of the second row of the corresponding triangle, are the following

$$
\begin{aligned}
A_{3,-5,4}^{(1,7,2,1)}(2,0) & =\left(\begin{array}{c}
-5 \\
4
\end{array}\right)^{2}\left(\begin{array}{l}
7 \\
2
\end{array}\right)^{1}=102900 \\
A_{3,-5,4}^{(1,7,2,1)}(2,1) & =\left(\begin{array}{c}
3-5 \\
4
\end{array}\right)^{2}\left(\begin{array}{c}
1+7 \\
2
\end{array}\right)-(8+2+1)\left(\begin{array}{c}
-5 \\
4
\end{array}\right)^{2}\left(\begin{array}{l}
7 \\
2
\end{array}\right)=-1131200 \\
A_{3,-5,4}^{(1,7,2,1)}(2,10) & =\left(\begin{array}{c}
3+5+4-1 \\
4
\end{array}\right)^{2}\left(\begin{array}{c}
1-7+2-1 \\
2
\end{array}\right)^{1}=1633500 .
\end{aligned}
$$

In fact, the second row of the triangle of generalized Eulerian numbers $A_{3,-5,4}^{(1,7,2,1)}(p, i)$ is

\begin{tabular}{|l|l|l|l|l|l|l|l|l|l|l|}
\hline 102900 & -1131200 & 5651800 & -16939955 & 33908380 & -45136850 & 58773550 & -21855275 & 13608790 & -7948490 & 1633500 \\
\hline
\end{tabular}

which means that the expansion of $\left(\begin{array}{c}3 n-5 \\ 4\end{array}\right)^{2}\left(\begin{array}{c}n+7 \\ 2\end{array}\right)$ is

$$
\begin{aligned}
& 102900\left(\begin{array}{c}
n+10 \\
10
\end{array}\right)-1131200\left(\begin{array}{c}
n+9 \\
10
\end{array}\right)+5651800\left(\begin{array}{c}
n+8 \\
10
\end{array}\right)-16939955\left(\begin{array}{c}
n+7 \\
10
\end{array}\right) \\
& +33908380\left(\begin{array}{c}
n+6 \\
10
\end{array}\right)-45136850\left(\begin{array}{c}
n+5 \\
10
\end{array}\right)+58772550\left(\begin{array}{c}
n+4 \\
10
\end{array}\right)-21855275\left(\begin{array}{c}
n+3 \\
10
\end{array}\right) \\
& +13608790\left(\begin{array}{c}
n+2 \\
10
\end{array}\right)-7948490\left(\begin{array}{c}
n+1 \\
10
\end{array}\right)+1633500\left(\begin{array}{c}
n \\
10
\end{array}\right) .
\end{aligned}
$$


Remark. From formula (25), it is clear that if $a=1$ and $b=0$ we have $A_{1,0, r}(p, i)=0$ for $i=0,1, \ldots, r-1$. Thus we can write (24) as $\left(\begin{array}{l}n \\ r\end{array}\right)^{p}=\sum_{i=r}^{r p} A_{1,0, r}(p, i)\left(\begin{array}{c}n+r p-i \\ r p\end{array}\right)$. In general, the value of the non-negative index $i$ before of which we have $A_{a, b, r}(p, i)=0$, depends on $a, b$ and $r$, as it is evident from formulas (28) and (29). However, in all cases when $a \neq 1$ or $b \neq 0$, we will consider the index $i$ beginning from $i=0$.

\subsection{Some generalized Eulerian numbers triangles}

We show now some concrete triangles of generalized Eulerian numbers. We will be using them as examples of the properties and results we will discuss for generalized Eulerian numbers in the rest of the article. We will write GENT\# to mean "generalized Eulerian numbers triangle number \#'.

\begin{tabular}{|c||c|c|c|c|c|c|c|c|c|c|c|c|c|}
\hline \hline \multicolumn{10}{|c|}{ GENT1: Generalized Eulerian Numbers $A_{2,-3,1}^{(3,2,3,2)}(p, i)}$. \\
\hline \hline \multicolumn{1}{|c|}{ Explicit Formula: $A_{2,-3,1}^{(3,2,3)}(p, i)=\sum_{j=0}^{i}(-1)^{j}\left(\begin{array}{c}p+7 \\
j\end{array}\right)(2(i-j)-3)^{p}\left(\begin{array}{c}3(i-j)+2 \\
3\end{array}\right)^{2}}$. \\
\hline$p \backslash i$ & 0 & 1 & 2 & 3 & 4 & 5 & 6 & 7 & 8 & 9 & $\cdots$ \\
\hline \hline 1 & 0 & -100 & 3936 & 53787 & 102488 & 41244 & 2760 & 5 & & & \\
\hline 2 & 0 & 100 & 2236 & 220401 & 1211671 & 1416076 & 396036 & 19375 & 25 & & $\cdots$ \\
\hline 3 & 0 & -100 & 4136 & 699215 & 9364370 & 25664255 & $\cdots$ & & & 125 & \\
\hline$\vdots$ & & $\vdots$ & & $\vdots$ & & $\vdots$ & & $\vdots$ & & $\vdots$ & \\
\hline
\end{tabular}

\begin{tabular}{|c|c|c|c|c|c|c|c|c|c|c|c|c|}
\hline \hline \multicolumn{1}{|c|}{ GENT2: Generalized Eulerian Numbers $A_{1,0,3}(p, i)}$. \\
\hline \hline \multicolumn{10}{|c|}{ Explicit Formula: $A_{1,0,3}(p, i)=\sum_{j=0}^{i}(-1)^{j}\left(\begin{array}{c}3 p+1 \\
j\end{array}\right)\left(\begin{array}{c}i-j \\
3\end{array}\right)^{p}}$. \\
\hline \multicolumn{1}{|c|}{$\left.\begin{array}{c}\text { Expansion: } \\
3\end{array}\right)^{p}=\sum_{i=3}^{3 p} A_{1,0,3}(p, i)}$. \\
\hline$p \backslash i$ & 3 & 4 & 5 & 6 & 7 & 8 & 9 & 10 & 11 & 12 & $\cdots$ \\
\hline \hline 1 & 1 & & & & & & & & & & \\
\hline 2 & 1 & 9 & 9 & 1 & & & & & & & $\cdots$ \\
\hline 3 & 1 & 54 & 405 & 760 & 405 & 54 & 1 & & & & \\
\hline 4 & 1 & 243 & 6750 & 49682 & 128124 & 128124 & 49682 & 6750 & 243 & 1 & $\cdots$ \\
\hline$\vdots$ & & $\vdots$ & & $\vdots$ & & $\vdots$ & & $\vdots$ & & $\vdots$ & \\
\hline
\end{tabular}




\begin{tabular}{|c|c|c|c|c|c|c|c|c|c|c|}
\hline \multicolumn{11}{|c|}{ GENT3: Generalized Eulerian Numbers $A_{3,2,2}(p, i)$. } \\
\hline \multicolumn{11}{|c|}{ Explicit Formula: $A_{3,2,2}(p, i)=\sum_{j=0}^{i}(-1)^{j}\left(\begin{array}{c}2 p+1 \\
j\end{array}\right)\left(\begin{array}{c}3(i-j)+2 \\
2\end{array}\right)^{p}}$. \\
\hline \multicolumn{11}{|c|}{ Expansion: $\left(\begin{array}{c}3 n+2 \\
2\end{array}\right)^{p}=\sum_{i=0}^{2 p} A_{3,2,2}(p, i)\left(\begin{array}{c}n+2 p-i \\
2 p\end{array}\right)$} \\
\hline$p \backslash i$ & 0 & 1 & 2 & 3 & 4 & 5 & 6 & 7 & 8 & $\ldots$ \\
\hline $\bar{~} 1$ & $\bar{~} 1$ & $\overline{77}$ & $\bar{~} 1$ & & & & & & & \\
\hline 2 & 1 & 95 & 294 & 95 & 1 & & & & & $\ldots$ \\
\hline 3 & 1 & 993 & 14973 & 33676 & 14973 & 993 & 1 & & & \\
\hline 4 & 1 & 9991 & 524692 & 3978637 & 7507078 & 3978637 & 524692 & 9991 & 1 & $\ldots$ \\
\hline$\vdots$ & & $\vdots$ & & $\vdots$ & & $\vdots$ & & $\vdots$ & & \\
\hline
\end{tabular}

\begin{tabular}{|c||c|c|c|c|c|c|c|c|c|c|c|}
\hline \hline \multicolumn{10}{|c|}{ GENT4: Generalized Eulerian Numbers $A_{1,4,3}(p, i)}$. \\
\hline \hline \multicolumn{10}{|c|}{ Explicit Formula: $A_{1,4,3}(p, i)=\sum_{j=0}^{i}(-1)^{j}\left(\begin{array}{c}3 p+1 \\
j\end{array}\right)\left(\begin{array}{c}i-j+4 \\
3\end{array}\right)^{p}}$. \\
\hline $\left.\begin{array}{c}|c| \\
3 p-i\end{array}\right)$. \\
\hline \hline 1 & 0 & 1 & 2 & 3 & 4 & 5 & 6 & 7 & 8 & 9 & $\cdots$ \\
\hline \hline 2 & 4 & -6 & 4 & -1 & & & & & & & \\
\hline 3 & 64 & -12 & 36 & -35 & 21 & -7 & 1 & & & & $\cdots$ \\
\hline$\vdots$ & & $\vdots$ & & $\vdots$ & & $\vdots$ & & $\vdots$ & & $\vdots$ & \\
\hline
\end{tabular}

The second rows of the generalized Eulerian numbers $A_{1,0, r}(p, i)$ triangles (for example GENT2), contain "known results", as we show now. The numbers $A_{1,0, r}(2, i)$ are

$$
A_{1,0, r}(2, i)=\sum_{j=0}^{i}(-1)^{j}\left(\begin{array}{c}
2 r+1 \\
j
\end{array}\right)\left(\begin{array}{c}
i-j \\
r
\end{array}\right)^{2}
$$

where $i=r, r+1, \ldots, 2 r$. By using the identity

$$
\sum_{j=0}^{k}(-1)^{j}\left(\begin{array}{c}
2 r+1 \\
j
\end{array}\right)\left(\begin{array}{c}
k+r-j \\
r
\end{array}\right)^{2}=\left(\begin{array}{l}
r \\
k
\end{array}\right)^{2}
$$

(formula (6.48) from Gould's book [13]), we see that the generalized Eulerian numbers $A_{1,0, r}(2, i)$ are squares of binomial coefficients. More precisely, for $k=0,1, \ldots, r$, we have

$$
A_{1,0, r}(2, k+r)=\sum_{j=0}^{k}(-1)^{j}\left(\begin{array}{c}
2 r+1 \\
j
\end{array}\right)\left(\begin{array}{c}
k+r-j \\
r
\end{array}\right)^{2}=\left(\begin{array}{l}
r \\
k
\end{array}\right)^{2}
$$

Thus, the corresponding expansion $\left(\begin{array}{l}n \\ r\end{array}\right)^{2}=\sum_{i=r}^{2 r} A_{1,0, r}(2, i)\left(\begin{array}{c}n+2 r-i \\ 2 r\end{array}\right)$ can be written as

$$
\left(\begin{array}{l}
n \\
r
\end{array}\right)^{2}=\sum_{k=0}^{r}\left(\begin{array}{l}
r \\
k
\end{array}\right)^{2}\left(\begin{array}{c}
n+r-k \\
2 r
\end{array}\right)
$$

which is identity (6.17) from Gould's book [13]. 


\section{Symmetries}

In this section we show some symmetries for generalized Eulerian numbers $A_{a, b, r}^{\left(\alpha_{s}, \beta_{s}, r_{s}, p_{s}\right)}(p, i)$.

Proposition 1. (a) The generalized Eulerian numbers $A_{a, b, r}(p, i)$ have the symmetry

$$
A_{a, b, r}(p, i)=A_{a, a-b+r-1, r}(p, r p-i),
$$

for $i=0,1, \ldots, r p$.

(b) The generalized Eulerian numbers $A_{a, b, r}^{\left(\alpha_{s}, \beta_{s}, r_{s}, p_{s}\right)}(p, i)$, where $r_{s}=2 \beta_{s}+1-\alpha_{s}, s=$ $2,3, \ldots$, l, have the symmetry

$$
A_{a, b, r}^{\left(\alpha_{s}, \beta_{s}, r_{s}, p_{s}\right)}(p, i)=A_{a, a-b+r-1, r}^{\left(\alpha_{s}, \beta_{s}, r_{s}, p_{s}\right)}(p, r p+\sigma-i),
$$

for $i=0,1, \ldots, r p+\sigma$.

Proof. We consider the expansion

$$
\begin{aligned}
\left(\begin{array}{c}
a n+b \\
r
\end{array}\right)^{p} \prod_{s=2}^{l}\left(\begin{array}{c}
\alpha_{s} n+\beta_{s} \\
r_{s}
\end{array}\right)^{p_{s}} & =\sum_{i=0}^{r p+\sigma} A_{a, b, r}^{\left(\alpha_{s}, \beta_{s}, r_{s}, p_{s}\right)}(p, i)\left(\begin{array}{c}
n+r p+\sigma-i \\
r p+\sigma
\end{array}\right) \\
& =\sum_{i=0}^{r p+\sigma} A_{a, b, r}^{\left(\alpha_{s}, \beta_{s}, r_{s}, p_{s}\right)}(p, r p+\sigma-i)\left(\begin{array}{c}
n+i \\
r p+\sigma
\end{array}\right) .
\end{aligned}
$$

In (42) replace $n$ by $-1-n$, to obtain

$$
\left(\begin{array}{c}
a(-1-n)+b \\
r
\end{array}\right)^{p} \prod_{s=2}^{l}\left(\begin{array}{c}
\alpha_{s}(-1-n)+\beta_{s} \\
r_{s}
\end{array}\right)^{p_{s}}=\sum_{i=0}^{r p+\sigma} A_{a, b, r}^{\left(\alpha_{s}, \beta_{s}, r_{s}, p_{s}\right)}(p, i)\left(\begin{array}{c}
-1-n+r p+\sigma-i \\
r p+\sigma
\end{array}\right),
$$

or

$$
\left(\begin{array}{c}
a n+a-b+r-1 \\
r
\end{array}\right)^{p} \prod_{s=2}^{l}\left(\begin{array}{c}
\alpha_{s} n+\alpha_{s}-\beta_{s}+r_{s}-1 \\
r_{s}
\end{array}\right)^{p_{s}}=\sum_{i=0}^{r p+\sigma} A_{a, b, r}^{\left(\alpha_{s}, \beta_{s}, r_{s}, p_{s}\right)}(p, i)\left(\begin{array}{c}
n+i \\
r p+\sigma
\end{array}\right) .
$$

Expression (43) says that

$$
\left(\begin{array}{c}
a n+a-b+r-1 \\
r
\end{array}\right)^{p} \prod_{s=2}^{l}\left(\begin{array}{c}
\alpha_{s} n+\beta_{s} \\
r_{s}
\end{array}\right)^{p_{s}}=\sum_{i=0}^{r p+\sigma} A_{a, a-b+r-1, r}^{\left(\alpha_{s}, \beta_{s}, r_{s}, p_{s}\right)}(p, r p+\sigma-i)\left(\begin{array}{c}
n+i \\
r p+\sigma
\end{array}\right) .
$$

(a) Set $r_{s}=0$ in (44) and (45) to conclude that, for $i=0,1, \ldots, r p$, we have that $A_{a, b, r}(p, i)$ is equal to $A_{a, a-b+r-1, r}(p, r p-i)$, as desired.

(b) If $r_{s}=2 \beta_{s}+1-\alpha_{s}, s=2,3, \ldots, l$, we can write (44) as

$$
\left(\begin{array}{c}
a n+a-b+r-1 \\
r
\end{array}\right)^{p} \prod_{s=2}^{l}\left(\begin{array}{c}
\alpha_{s} n+\beta_{s} \\
r_{s}
\end{array}\right)^{p_{s}}=\sum_{i=0}^{r p+\sigma} A_{a, b, r}^{\left(\alpha_{s}, \beta_{s}, r_{s}, p_{s}\right)}(p, i)\left(\begin{array}{c}
n+i \\
r p+\sigma
\end{array}\right)
$$

and again, comparing (45) with (46), we obtain the desired conclusion (41). 
What Proposition 1 says is that if we have

$$
\left(\begin{array}{c}
a n+b \\
r
\end{array}\right)^{p} \prod_{s=2}^{l}\left(\begin{array}{c}
\alpha_{s} n+\beta_{s} \\
r_{s}
\end{array}\right)^{p_{s}}=\sum_{i=0}^{r p+\sigma} A_{a, b, r}^{\left(\alpha_{s}, \beta_{s}, r_{s}, p_{s}\right)}(p, i)\left(\begin{array}{c}
n+r p+\sigma-i \\
r p+\sigma
\end{array}\right)
$$

then, in any of the cases: (i) $r_{s}=0, s=2,3, \ldots, l$, or (ii) $r_{s}=2 \beta_{s}+1-\alpha_{s}, s=2,3, \ldots, l$, we also have

$$
\begin{aligned}
& \left(\begin{array}{c}
a n+a-b+r-1 \\
r
\end{array}\right)^{p} \prod_{s=2}^{l}\left(\begin{array}{c}
\alpha_{s} n+\beta_{s} \\
r_{s}
\end{array}\right)^{p_{s}} \\
= & \sum_{i=0}^{r p+\sigma} A_{a, b, r}^{\left(\alpha_{s}, \beta_{s}, r_{s}, p_{s}\right)}(p, r p+\sigma-i)\left(\begin{array}{c}
n+r p+\sigma-i \\
r p+\sigma
\end{array}\right) .
\end{aligned}
$$

Two examples from Proposition 1 are the following, (we write together expressions (47) and (48))

$$
\begin{gathered}
(5 n-1)^{2}=\left(\begin{array}{c}
n+2 \\
2
\end{array}\right)+13\left(\begin{array}{c}
n+1 \\
2
\end{array}\right)+36\left(\begin{array}{c}
n \\
2
\end{array}\right), \\
(5 n+6)^{2}=36\left(\begin{array}{c}
n+2 \\
2
\end{array}\right)+13\left(\begin{array}{c}
n+1 \\
2
\end{array}\right)+\left(\begin{array}{c}
n \\
2
\end{array}\right) . \\
(3 n-1)(2 n+1)\left(\begin{array}{c}
4 n+3 \\
3
\end{array}\right)=-\left(\begin{array}{c}
n+5 \\
5
\end{array}\right)+216\left(\begin{array}{c}
n+4 \\
5
\end{array}\right)+2850\left(\begin{array}{c}
n+3 \\
5
\end{array}\right)+3900\left(\begin{array}{c}
n+2 \\
5
\end{array}\right)+711\left(\begin{array}{c}
n+1 \\
5
\end{array}\right)+4\left(\begin{array}{c}
n \\
5
\end{array}\right), \\
(3 n+4)(2 n+1)\left(\begin{array}{c}
4 n+3 \\
3
\end{array}\right)=4\left(\begin{array}{c}
n+5 \\
5
\end{array}\right)+711\left(\begin{array}{c}
n+4 \\
5
\end{array}\right)+3900\left(\begin{array}{c}
n+3 \\
5
\end{array}\right)+2850\left(\begin{array}{c}
n+2 \\
5
\end{array}\right)+216\left(\begin{array}{c}
n+1 \\
5
\end{array}\right)-\left(\begin{array}{c}
n \\
5
\end{array}\right) .
\end{gathered}
$$

Observe that in the case

$$
\begin{aligned}
& \left(\begin{array}{c}
3 n+2 \\
2
\end{array}\right)\left(\begin{array}{c}
4 n+3 \\
3
\end{array}\right) \\
= & \left(\begin{array}{c}
n+5 \\
5
\end{array}\right)+344\left(\begin{array}{c}
n+4 \\
5
\end{array}\right)+2535\left(\begin{array}{c}
n+3 \\
5
\end{array}\right)+2535\left(\begin{array}{c}
n+2 \\
5
\end{array}\right)+344\left(\begin{array}{c}
n+1 \\
5
\end{array}\right)+\left(\begin{array}{c}
n \\
5
\end{array}\right),
\end{aligned}
$$

besides the hypothesis $3=r_{2}=2 \beta_{2}+1-\alpha_{2}=2(3)+1-4$, we have also $2=r=$ $2 b+1-a=2(2)+1-3$. Thus, expressions (47) and (48) are identical. That is, in this case we have the symmetry $A_{3,2,2}^{(4,3,3,1)}(1, i)=A_{3,2,2}^{(4,3,3,1)}(1,5-i), i=0,1, \ldots, 5$. Observe also that if $r=2 b+1-a$, expression (40) looks as $A_{a, b, r}(p, i)=A_{a, b, r}(p, r p-i), i=0,1, \ldots, r p$. This is the case of generalized Eulerian numbers $A_{3,2,2}(p, i)$ (see GENT3 in Subsection 2.1). Yet another particular case from (40) is $A_{2,1, r}(p, i)=A_{2, r, r}(p, r p-i), i=0,1, \ldots, r p$. That is, we have

$$
\begin{aligned}
& \left(\begin{array}{c}
2 n+1 \\
r
\end{array}\right)^{p}=\sum_{i=0}^{r p} A_{2,1, r}(p, i)\left(\begin{array}{c}
n+r p-i \\
r p
\end{array}\right)=\sum_{i=0}^{r p} A_{2, r, r}(p, i)\left(\begin{array}{c}
n+i \\
r p
\end{array}\right) \\
& \left(\begin{array}{c}
2 n+r \\
r
\end{array}\right)^{p}=\sum_{i=0}^{r p} A_{2, r, r}(p, i)\left(\begin{array}{c}
n+r p-i \\
r p
\end{array}\right)=\sum_{i=0}^{r p} A_{2,1, r}(p, i)\left(\begin{array}{c}
n+i \\
r p
\end{array}\right) .
\end{aligned}
$$

For example

$$
\begin{aligned}
& \left(\begin{array}{c}
2 n+1 \\
6
\end{array}\right)=7\left(\begin{array}{c}
n+3 \\
6
\end{array}\right)+35\left(\begin{array}{c}
n+2 \\
6
\end{array}\right)+21\left(\begin{array}{c}
n+1 \\
6
\end{array}\right)+\left(\begin{array}{c}
n \\
6
\end{array}\right) \\
& \left(\begin{array}{c}
2 n+6 \\
6
\end{array}\right)=\left(\begin{array}{c}
n+6 \\
6
\end{array}\right)+21\left(\begin{array}{c}
n+5 \\
6
\end{array}\right)+35\left(\begin{array}{c}
n+4 \\
6
\end{array}\right)+7\left(\begin{array}{c}
n+3 \\
6
\end{array}\right) .
\end{aligned}
$$


As a final example, we show the following curious situation

$$
\begin{aligned}
\left(\begin{array}{c}
3 n \\
2
\end{array}\right)^{2} & =9\left(\begin{array}{c}
n+3 \\
4
\end{array}\right)+180\left(\begin{array}{c}
n+2 \\
4
\end{array}\right)+261\left(\begin{array}{c}
n+1 \\
4
\end{array}\right)+36\left(\begin{array}{l}
n \\
4
\end{array}\right) \\
\left(\begin{array}{c}
3 n+1 \\
2
\end{array}\right)^{2} & =36\left(\begin{array}{c}
n+3 \\
4
\end{array}\right)+261\left(\begin{array}{c}
n+2 \\
4
\end{array}\right)+180\left(\begin{array}{c}
n+1 \\
4
\end{array}\right)+9\left(\begin{array}{c}
n \\
4
\end{array}\right) \\
\left(\begin{array}{c}
3 n+3 \\
2
\end{array}\right)^{2} & =9\left(\begin{array}{c}
n+4 \\
4
\end{array}\right)+180\left(\begin{array}{c}
n+3 \\
4
\end{array}\right)+261\left(\begin{array}{c}
n+2 \\
4
\end{array}\right)+36\left(\begin{array}{c}
n+1 \\
4
\end{array}\right) \\
\left(\begin{array}{c}
3 n+4 \\
2
\end{array}\right)^{2} & =36\left(\begin{array}{c}
n+4 \\
4
\end{array}\right)+261\left(\begin{array}{c}
n+3 \\
4
\end{array}\right)+180\left(\begin{array}{c}
n+2 \\
4
\end{array}\right)+9\left(\begin{array}{c}
n+1 \\
4
\end{array}\right) .
\end{aligned}
$$

The symmetry (40) says that $A_{3, b, 2}(2, i)=A_{3,4-b, 2}(2,4-i), 0 \leq i \leq 4$. Thus (with $b=0$ ) we have $A_{3,0,2}(2, i)=A_{3,4,2}(2,4-i)$, which explains the relation between (51) and (54). Also (with $b=1$ ) we have $A_{3,1,2}(2, i)=A_{3,3,2}(2,4-i)$, which explains the relation between (52) and (53). The relations $(51) \leftrightarrow(52)$ and $(53) \leftrightarrow(54)$, are 'part of the curiosity' of this example. In fact, by replacing $n$ by $-n$ in (51) we obtain (52). If in (53) we replace $n$ by $-n$, we obtain

$$
\left(\begin{array}{c}
3 n-2 \\
2
\end{array}\right)^{2}=9\left(\begin{array}{c}
n-1 \\
4
\end{array}\right)+180\left(\begin{array}{l}
n \\
4
\end{array}\right)+261\left(\begin{array}{c}
n+1 \\
4
\end{array}\right)+36\left(\begin{array}{c}
n+2 \\
4
\end{array}\right) .
$$

Now replace $n$ by $n+2$ in (55) to obtain (54).

In the case $a=1, b=0$, the symmetry (40) says that $A_{1,0, r}(p, r p-i)=A_{1, r, r}(p, i)$. But plainly we have, for $i=0,1, \ldots, r p$, that

$$
A_{1, r, r}(p, i)=A_{1,0, r}(p, r+i),
$$

Thus, the symmetry (40) can be written in this case as

$$
A_{1,0, r}(p, r(p+1)-i)=A_{1,0, r}(p, i) .
$$

(See Lemma 2 in [21].) The triangle GENT2 in Subsection 2.1 shows the symmetry (57). Of course, the known case (2) of the standard Eulerian numbers $A_{1,0,1}(p, i)$ is also included in (57).

It is not difficult to see that $A_{1, r, r}(p, i)=0$ for $i=r(p-1)+1, \ldots, r p$. Then (56) is valid for $i=0,1, \ldots, r(p-1)$. For example, we have

$$
\left(\begin{array}{l}
n \\
5
\end{array}\right)^{2}=\sum_{i=0}^{5} c_{i}\left(\begin{array}{c}
n+5-i \\
10
\end{array}\right) \quad \text { and } \quad\left(\begin{array}{c}
n+5 \\
5
\end{array}\right)^{2}=\sum_{i=0}^{5} c_{i}\left(\begin{array}{c}
n+10-i \\
10
\end{array}\right)
$$

where $c_{0}=c_{5}=1, c_{1}=c_{4}=25$ and $c_{2}=c_{3}=100$.

Next we consider the case $a=2$ and $b=1$.

Proposition 2. For odd $r \in \mathbb{N}$, the generalized Eulerian numbers $A_{2,1, r}(p, i)$ satisfy the symmetry

$$
A_{2,1, r}\left(p, i+\frac{r-1}{2}\right)=A_{2,1, r}(p, r p-i)
$$

where $i=0,1, \ldots, r p-\frac{r-1}{2}$. 
Proof. The generalized Eulerian numbers (from (26), with $a=2, b=1$ )

$$
A_{2,1, r}(p, i)=\sum_{j=0}^{i}(-1)^{j}\left(\begin{array}{c}
r p+1 \\
j
\end{array}\right)\left(\begin{array}{c}
2(i-j)+1 \\
r
\end{array}\right)^{p},
$$

are such that $A_{2,1, r}(p, i)=0$ for $i=0,1, \ldots, \frac{r-1}{2}-1$. Thus, we can write

$$
\left(\begin{array}{c}
2 n+1 \\
r
\end{array}\right)^{p}=\sum_{i=\frac{r-1}{2}}^{r p} A_{2,1, r}(p, i)\left(\begin{array}{c}
n+r p-i \\
r p
\end{array}\right)
$$

or

$$
\begin{aligned}
\left(\begin{array}{c}
2 n+1 \\
r
\end{array}\right)^{p} & =\sum_{i=0}^{r p-\frac{r-1}{2}} A_{2,1, r}\left(p, i+\frac{r-1}{2}\right)\left(\begin{array}{c}
n+r p-i-\frac{r-1}{2} \\
r p
\end{array}\right) \\
& =\sum_{i=0}^{r p-\frac{r-1}{2}} A_{2,1, r}(p, r p-i)\left(\begin{array}{c}
n+i \\
r p
\end{array}\right) .
\end{aligned}
$$

In (59) replace $n$ by $\frac{r-1}{2}-1-n$ to obtain

$$
\left(\begin{array}{c}
r-2-2 n \\
r
\end{array}\right)^{p}=\sum_{i=0}^{r p-\frac{r-1}{2}} A_{2,1, r}\left(p, i+\frac{r-1}{2}\right)\left(\begin{array}{c}
-n-1+r p-i \\
r p
\end{array}\right)
$$

that is

$$
\left(\begin{array}{c}
2 n+1 \\
r
\end{array}\right)^{p}=\sum_{i=0}^{r p-\frac{r-1}{2}} A_{2,1, r}\left(p, i+\frac{r-1}{2}\right)\left(\begin{array}{c}
n+i \\
r p
\end{array}\right)
$$

Comparing (60) with (61) we obtain the desired conclusion.

In particular (the case $r=1$ of (58)), we have that $A_{2,1,1}(p, i)=A_{2,1,1}(p, p-i)$ (which can also be concluded from (49) and (50)). An example of this particular case (with $p=4$ ) is the expansion

$$
(2 n+1)^{4}=\left(\begin{array}{c}
n+4 \\
4
\end{array}\right)+76\left(\begin{array}{c}
n+3 \\
4
\end{array}\right)+230\left(\begin{array}{c}
n+2 \\
4
\end{array}\right)+76\left(\begin{array}{c}
n+1 \\
4
\end{array}\right)+\left(\begin{array}{l}
n \\
4
\end{array}\right) .
$$

An example of (58), with $r=3$ and $p=2$, is

$$
\left(\begin{array}{c}
2 n+1 \\
3
\end{array}\right)^{2}=\left(\begin{array}{c}
n+5 \\
6
\end{array}\right)+93\left(\begin{array}{c}
n+4 \\
6
\end{array}\right)+546\left(\begin{array}{c}
n+3 \\
6
\end{array}\right)+546\left(\begin{array}{c}
n+2 \\
6
\end{array}\right)+93\left(\begin{array}{c}
n+1 \\
6
\end{array}\right)+\left(\begin{array}{c}
n \\
6
\end{array}\right) .
$$

Finally, we comment that for the generalized Eulerian numbers $A_{2,1,3}^{(1,0,1, q)}(p, i)$, where $q$ is any non-negative integer given, we have the symmetry

$$
A_{2,1,3}^{(1,0,1, q)}(p, i+1)=A_{2,1,3}^{(1,0,1, q)}(p, 3 p+q-i),
$$

where $i=0,1, \ldots, 3 p+q$. The proof of this fact is similar to the proof of Proposition 2, and we leave it to the reader. An example (with $q=2$ and $p=1$ ) is

$$
n^{2}\left(\begin{array}{c}
2 n+1 \\
3
\end{array}\right)=\left(\begin{array}{c}
n+4 \\
5
\end{array}\right)+34\left(\begin{array}{c}
n+3 \\
5
\end{array}\right)+90\left(\begin{array}{c}
n+2 \\
5
\end{array}\right)+34\left(\begin{array}{c}
n+1 \\
5
\end{array}\right)+\left(\begin{array}{l}
n \\
5
\end{array}\right) .
$$




\section{Recurrence}

Let us see the case $r=1$. We want to relate the generalized Eulerian number $A_{a, b, 1}^{\left(\alpha_{s}, \beta_{s}, r_{s}, p_{s}\right)}(p, i)$ in the $p$-th row and $i$-th column $(i=0,1, \ldots, p+\sigma)$ of the corresponding triangle of generalized Eulerian numbers, with "some" of the generalized Eulerian numbers $A_{a, b, 1}^{\left(\alpha_{s}, \beta_{s}, r_{s}, p_{s}\right)}(p-1, *)$ from the $(p-1)$-th row.

Our strategy will be to obtain the $Z$-transform of the sequence $(a n+b)^{p} \prod_{s=2}^{l}\left(\begin{array}{c}\alpha_{s} n+\beta_{s} \\ r_{s}\end{array}\right)^{p_{s}}$, namely

$$
\mathcal{Z}\left((a n+b)^{p} \prod_{s=2}^{l}\left(\begin{array}{c}
\alpha_{s} n+\beta_{s} \\
r_{s}
\end{array}\right)^{p_{s}}\right)=\frac{z \sum_{i=0}^{p+\sigma} A_{a, b, 1}^{\left(\alpha_{s}, \beta_{s}, r_{s}, p_{s}\right)}(p, i) z^{p+\sigma-i}}{(z-1)^{p+\sigma+1}}
$$

(see (26) and (27)), and relate it with the $Z$-transform of the sequence $(a n+b)^{p-1} \prod_{s=2}^{l}\left(\begin{array}{c}\alpha_{s} n+\beta_{s} \\ r_{s}\end{array}\right)^{p_{s}}$. The beginning is easy: we use (10) and (65) with $p$ replaced by $p-1$, to write (65) as

$$
\begin{aligned}
& \mathcal{Z}\left((a n+b)^{p} \prod_{s=2}^{l}\left(\begin{array}{c}
\alpha_{s} n+\beta_{s} \\
r_{s}
\end{array}\right)^{p_{s}}\right) \\
= & a \mathcal{Z}\left(n(a n+b)^{p-1} \prod_{s=2}^{l}\left(\begin{array}{c}
\alpha_{s} n+\beta_{s} \\
r_{s}
\end{array}\right)^{p_{s}}\right)+b \mathcal{Z}\left((a n+b)^{p-1} \prod_{s=2}^{l}\left(\begin{array}{c}
\alpha_{s} n+\beta_{s} \\
r_{s}
\end{array}\right)^{p_{s}}\right) \\
= & -a z \frac{d}{d z} \frac{z \sum_{i=0}^{p-1+\sigma} A_{a, b, 1}^{\left(\alpha_{s}, \beta_{s}, r_{s}, p_{s}\right)}(p-1, i) z^{p-1+\sigma-i}}{(z-1)^{p-1+\sigma+1}}+b \frac{z \sum_{i=0}^{p-1+\sigma} A_{a, b, 1}^{\left(\alpha_{s}, \beta_{s}, r_{s}, p_{s}\right)}(p-1, i) z^{p-1+\sigma-i}}{(z-1)^{p-1+\sigma+1}} .
\end{aligned}
$$

After performing the derivative and some simplifications we get

$$
\begin{aligned}
\mathcal{Z}\left((a n+b)^{p} \prod_{s=2}^{l}\left(\begin{array}{c}
\alpha_{s} n+\beta_{s} \\
r_{s}
\end{array}\right)^{p_{s}}\right) \\
=\frac{z \sum_{i=0}^{p+\sigma}\left(\begin{array}{c}
(a(p+\sigma-i+1)-b) A_{a, b, 1}^{\left(\alpha_{s}, \beta_{s}, r_{s}, p_{s}\right)}(p-1, i-1) \\
+(a i+b) A_{a, b, 1}^{\left(\alpha, \beta, p_{1}\right)}(p-1, i)
\end{array}\right) z^{p+\sigma-i}}{(z-1)^{p+\sigma+1}} .
\end{aligned}
$$

From (65) and (66), we obtain the recurrence

$$
\begin{aligned}
& A_{a, b, 1}^{\left(\alpha_{s}, \beta_{s}, r_{s}, p_{s}\right)}(p, i) \\
= & (a i+b) A_{a, b, 1}^{\left(\alpha_{s}, \beta_{s}, r_{s}, p_{s}\right)}(p-1, i)+(a(p+\sigma-i+1)-b) A_{a, b, 1}^{\left(\alpha_{s}, \beta_{s}, r_{s}, p_{s}\right)}(p-1, i-1) .
\end{aligned}
$$

Formula (67) contains the recurrence

$$
A_{a, b, 1}(p, i)=(a i+b) A_{a, b, 1}(p-1, i)+(a(p-i+1)-b) A_{a, b, 1}(p-1, i-1),
$$

(see formula 20 in [25]), and in turn formula (68) contains the case of the well-known recurrence (3) for the standard Eulerian numbers $A_{1,0,1}(p, i)$. 
For example, for the generalized Eulerian numbers $A_{2,-3,1}^{(3,2,3,2)}(p, i)$ we have the recurrence (according to (67))

$$
A_{2,-3,1}^{(3,2,3,2)}(p, i)=(2 i-3) A_{2,-3,1}^{(3,2,3,2)}(p-1, i)+(2(p-i)+17) A_{2,-3,1}^{(3,2,3,2)}(p-1, i-1) .
$$

If $p=3$ and $i=5$ we have (see GENT1 in Subsection 2.1)

$A_{2,-3,1}^{(3,2,3,2)}(3,5)=7 A_{2,-3,1}^{(3,2,3,2)}(2,5)+13 A_{2,-3,1}^{(3,2,3,2)}(2,4)=7(1416076)+13(1211671)=25664255$.

We can mimic the previous procedure in order to obtain the following recurrence in the case $r=2$ (we write $\sigma$ for $\sigma$ )

$$
\begin{aligned}
& A_{a, b, 2}^{\left(\alpha_{s}, \beta_{s}, r_{s}, p_{s}\right)}(p, i) \\
= & \frac{1}{2}(a i+b)(a i+b-1) A_{a, b, 2}^{\left(\alpha_{s}, \beta_{s}, r_{s}, p_{s}\right)}(p-1, i) \\
& +\frac{1}{2}\left\{\begin{array}{c}
-2 a^{2} i^{2}+2 a i(a-2 b+a(2 p+\sigma)+1) \\
+2 b-a+2 a b-a(2 p+\sigma)+2 a b(2 p+\sigma) \\
-a^{2}-2 b^{2}-a^{2}(2 p+\sigma)
\end{array}\right\} A_{a, b, 2}^{\left(\alpha_{s}, \beta_{s}, r_{s}, p_{s}\right)}(p-1, i-1) \\
& +\frac{1}{2}(a(i-2 p-\sigma-1)+b)(a(i-2 p-\sigma-1)+b-1) A_{a, b, 2}^{\left(\alpha_{s}, \beta_{s}, r_{s}, p_{s}\right)}(p-1, i-2) .
\end{aligned}
$$

For example, the recurrence for the generalized Eulerian numbers $A_{3,2,2}(p, i)$ is

$$
\begin{aligned}
A_{3,2,2}(p, i)= & \left(\begin{array}{c}
3 i+2 \\
2
\end{array}\right) A_{3,2,2}(p-1, i)+\left(-9 i^{2}+18 i p-2\right) A_{3,2,2}(p-1, i-1) \\
& +\left(\begin{array}{c}
3(i-2 p)-1 \\
2
\end{array}\right) A_{3,2,2}(p-1, i-2) .
\end{aligned}
$$

If $p=4$ and $i=5$ we have (see GENT3 in Subsection 2.1)

$$
\begin{aligned}
A_{3,2,2}(4,5) & =\left(\begin{array}{c}
17 \\
2
\end{array}\right) A_{3,2,2}(3,5)+133 A_{3,2,2}(3,4)+\left(\begin{array}{c}
-10 \\
2
\end{array}\right) A_{3,2,2}(3,3) \\
& =136(993)+133(14973)+55(33676)=3978637
\end{aligned}
$$

Recurrence (70) does not offer any motivation to continue trying the next particular cases $r=3,4, \ldots$ (seeking a conjecture for the general case). The good news come from the fact that if $a=1$, we have a nice general recurrence.

Proposition 3. The recurrence for the generalized Eulerian numbers $A_{1, b, r}^{\left(\alpha_{s}, \beta_{s}, r_{s}, p_{s}\right)}(p, i)$ is given by

$$
A_{1, b, r}^{\left(\alpha_{s}, \beta_{s}, r_{s}, p_{s}\right)}(p, i)=\sum_{k=0}^{r}\left(\begin{array}{c}
i-k+b \\
r-k
\end{array}\right)\left(\begin{array}{c}
r p+\sigma+k-i-b \\
k
\end{array}\right) A_{1, b, r}^{\left(\alpha_{s}, \beta_{s}, r_{s}, p_{s}\right)}(p-1, i-k),
$$

where $i=0,1, \ldots, r p+\sigma$. 
Proof. From (22) and (23) we can write

$$
\begin{aligned}
& \sum_{i=0}^{r p+\sigma} A_{1, b, r}^{\left(\alpha_{s}, \beta_{s}, r_{s}, p_{s}\right)}(p, i)\left(\begin{array}{c}
n+r p+\sigma-i \\
r p+\sigma
\end{array}\right) \\
= & \left(\begin{array}{c}
n+b \\
r
\end{array}\right)\left(\begin{array}{c}
n+b \\
r
\end{array}\right) \prod_{s=2}^{p-1}\left(\begin{array}{c}
\alpha_{s} n+\beta_{s} \\
r_{s}
\end{array}\right) p^{p_{s}} \\
= & \left(\begin{array}{c}
n+b \\
r
\end{array}\right) \sum_{i=0}^{r(p-1)+\sigma} A_{a, b, r}^{\left(\alpha_{s}, \beta_{s}, r_{s}, p_{s}\right)}(p-1, i)\left(\begin{array}{c}
n+r(p-1)+\sigma-i \\
r(p-1)+\sigma
\end{array}\right) .
\end{aligned}
$$

Thus, to prove (72) we have to prove that

$$
\begin{aligned}
& \sum_{i=0}^{r p+\sigma} \sum_{k=0}^{r}\left(\begin{array}{c}
i-k+b \\
r-k
\end{array}\right)\left(\begin{array}{c}
r p+\sigma+k-i-b \\
k
\end{array}\right) A_{1, b, r}^{\left(\alpha_{s}, \beta_{s}, r_{s}, p_{s}\right)}(p-1, i-k)\left(\begin{array}{c}
n+r p+\sigma-i \\
r p+\sigma
\end{array}\right) \\
= & \left(\begin{array}{c}
n+b \\
r
\end{array}\right) \sum_{i=0}^{r(p-1)+\sigma} A_{1, b, r}^{\left(\alpha_{s}, \beta_{s}, r_{s}, p_{s}\right)}(p-1, i)\left(\begin{array}{c}
n+r(p-1)+\sigma-i \\
r(p-1)+\sigma
\end{array}\right) .
\end{aligned}
$$

In the expression of the left-hand side of (74), we introduce the new index $I=i-k$, which runs from $I=0$ up to $I=r(p-1)+\sigma$. Then we write (74) as

$$
\begin{aligned}
& \sum_{i=0}^{r p+\sigma} \sum_{k=0}^{r}\left(\begin{array}{c}
i-k+b \\
r-k
\end{array}\right)\left(\begin{array}{c}
r p+\sigma+k-i-b \\
k
\end{array}\right) A_{1, b, r}^{\left(\alpha_{s}, \beta_{s}, r_{s}, p_{s}\right)}(p-1, i-k)\left(\begin{array}{c}
n+r p+\sigma-i \\
r p+\sigma
\end{array}\right) \\
= & \sum_{I=0}^{r(p-1)+\sigma} \sum_{k=0}^{r}\left(\begin{array}{c}
I+b \\
r-k
\end{array}\right)\left(\begin{array}{c}
r p+\sigma-I-b \\
k
\end{array}\right)\left(\begin{array}{c}
n+r p+\sigma-I-k \\
r p+\sigma
\end{array}\right) A_{1, b, r}^{\left(\alpha_{s}, \beta_{s}, r_{s}, p_{s}\right)}(p-1, I) .
\end{aligned}
$$

The proof ends if we show that

$$
\begin{aligned}
& \sum_{k=0}^{r}\left(\begin{array}{c}
I+b \\
r-k
\end{array}\right)\left(\begin{array}{c}
r p+\sigma-I-b \\
k
\end{array}\right)\left(\begin{array}{c}
n+r p+\sigma-I-k \\
r p+\sigma
\end{array}\right) \\
= & \left(\begin{array}{c}
n+b \\
r
\end{array}\right)\left(\begin{array}{c}
n+r(p-1)+\sigma-I \\
r(p-1)+\sigma
\end{array}\right) .
\end{aligned}
$$

If in identity (6.43) of Gould's book [13], namely

$$
\sum_{\kappa}\left(\begin{array}{l}
\beta \\
\kappa
\end{array}\right)\left(\begin{array}{c}
\gamma \\
\delta-\kappa
\end{array}\right)\left(\begin{array}{l}
\alpha+\kappa \\
\beta+\gamma
\end{array}\right)=\left(\begin{array}{c}
\alpha \\
\beta+\gamma-\delta
\end{array}\right)\left(\begin{array}{c}
\alpha-\gamma+\delta \\
\delta
\end{array}\right),
$$

we set $\alpha=-n+I-1, \beta=r p+\sigma-I-b, \gamma=I+b, \delta=r$ and $\kappa=k$, we obtain (75). Then the proof is complete.

In particular we have from (72) (with $i=0$ ), that $A_{1, b, r}^{\left(\alpha_{s}, \beta_{s}, r_{s}, p_{s}\right)}(p, 0)=\left(\begin{array}{l}b \\ r\end{array}\right) A_{1, b, r}^{\left(\alpha_{s}, \beta_{s}, r_{s}, p_{s}\right)}(p-1,0)$ (see (28)). If we set $i=1$ in (72) and use (28) and (29), we obtain the following formula (with flavor of Pascal's triangle recurrence)

$$
(r p+\sigma+1)\left(\begin{array}{l}
b \\
r
\end{array}\right)+(r p+\sigma-b)\left(\begin{array}{c}
b \\
r-1
\end{array}\right)=(r(p-1)+\sigma+1)\left(\begin{array}{c}
b+1 \\
r
\end{array}\right) .
$$


Let us see a concrete numerical example from the recurrence (72). The generalized Eulerian numbers $A_{1,0,3}(p, i)$ have the recurrence

$$
\begin{aligned}
A_{1,0,3}(p, i)= & \left(\begin{array}{l}
i \\
3
\end{array}\right) A_{1,0,3}(p-1, i)+\left(\begin{array}{c}
i-1 \\
2
\end{array}\right)\left(\begin{array}{c}
3 p+1-i \\
1
\end{array}\right) A_{1,0,3}(p-1, i-1) \\
& +\left(\begin{array}{c}
i-2 \\
1
\end{array}\right)\left(\begin{array}{c}
3 p+2-i \\
2
\end{array}\right) A_{1,0,3}(p-1, i-2) \\
& +\left(\begin{array}{c}
3 p+3-i \\
3
\end{array}\right) A_{1,0,3}(p-1, i-3) .
\end{aligned}
$$

If in (76) we set $p=4, i=7$ (see GENT2 in Subsection 2.1), then we get

$$
\begin{aligned}
& A_{1,0,3}(4,7) \\
= & \left(\begin{array}{l}
7 \\
3
\end{array}\right) A_{1,0,3}(3,7)+\left(\begin{array}{l}
6 \\
2
\end{array}\right)\left(\begin{array}{l}
6 \\
1
\end{array}\right) A_{1,0,3}(3,6)+\left(\begin{array}{l}
5 \\
1
\end{array}\right)\left(\begin{array}{l}
7 \\
2
\end{array}\right) A_{1,0,3}(3,5)+\left(\begin{array}{l}
8 \\
3
\end{array}\right) A_{1,0,3}(3,4) \\
= & \left(\begin{array}{l}
7 \\
3
\end{array}\right)(405)+\left(\begin{array}{l}
6 \\
2
\end{array}\right)\left(\begin{array}{l}
6 \\
1
\end{array}\right)(760)+\left(\begin{array}{l}
5 \\
1
\end{array}\right)\left(\begin{array}{l}
7 \\
2
\end{array}\right)(405)+\left(\begin{array}{l}
8 \\
3
\end{array}\right)(54) \\
= & 128124 .
\end{aligned}
$$

Finally, observe that the generalized Eulerian numbers $A_{1,0, r}(3, i)$ (from the third row of the corresponding triangle), can be written in terms of squares of binomial coefficients (in terms of the generalized Eulerian numbers $A_{1,0, r}(2, i)$ (see (38)). In this case the recurrence (72) gives us the explicit formula

$$
A_{1,0, r}(3, i)=\sum_{k=0}^{r}\left(\begin{array}{c}
i-k \\
i-r
\end{array}\right)\left(\begin{array}{c}
3 r+k-i \\
k
\end{array}\right)\left(\begin{array}{c}
r \\
2 r+k-i
\end{array}\right)^{2} .
$$

That is, for $i=r, \ldots, 3 r$, we have the identity

$$
\sum_{j=0}^{i}(-1)^{j}\left(\begin{array}{c}
3 r+1 \\
j
\end{array}\right)\left(\begin{array}{c}
i-j \\
r
\end{array}\right)^{3}=\sum_{k=0}^{r}\left(\begin{array}{c}
i-k \\
i-r
\end{array}\right)\left(\begin{array}{c}
3 r+k-i \\
k
\end{array}\right)\left(\begin{array}{c}
r \\
2 r+k-i
\end{array}\right)^{2} .
$$

\section{Row sums}

Let us consider the generalized Eulerian numbers $A_{a, b, r}^{\left(\alpha_{s}, \beta_{s}, r_{s}, p_{s}\right)}(p, i)$ involved in the expansion (22). We want an expression for the sum of the elements of the $p$-th row of the corresponding triangle of generalized Eulerian numbers, namely

$$
\sum_{i=0}^{r p+\sigma} A_{a, b, r}^{\left(\alpha_{s}, \beta_{s}, r_{s}, p_{s}\right)}(p, i)
$$

The result is given in the following Proposition.

Proposition 4. The sum of the generalized Eulerian numbers $A_{a, b, r}^{\left(\alpha_{s}, \beta_{s}, r_{s}, p_{s}\right)}(p, i), i=0,1, \ldots, r p+$ $\sigma$, is given by

$$
\sum_{i=0}^{r p+\sigma} A_{a, b, r}^{\left(\alpha_{s}, \beta_{s}, r_{s}, p_{s}\right)}(p, i)=(r p+\sigma) ! \frac{a^{r p}}{(r !)^{p}} \prod_{s=2}^{l} \frac{\alpha_{s}^{r_{s} p_{s}}}{\left(r_{s} !\right)^{p_{s}}} .
$$

(Observe that this result does not depend on b nor $\beta_{s}$.) 
Proof. The coefficient of $n^{r p+\sigma}$ in $\left(\begin{array}{c}a n+b \\ r\end{array}\right)^{p} \prod_{s=2}^{l}\left(\begin{array}{c}\alpha_{s} n+\beta_{s} \\ r_{s}\end{array}\right)^{p_{s}}$ (the left-hand side of (22)), is

$$
\frac{a^{r p}}{(r !)^{p}} \prod_{s=2}^{l} \frac{\alpha_{s}^{r_{s} p_{s}}}{\left(r_{s} !\right)^{p_{s}}}
$$

The coefficient of $n^{r p+\sigma}$ in $\sum_{i=0}^{r p+\sigma} A_{a, b, r}^{\left(\alpha_{s}, \beta_{s}, r_{s}, p_{s}\right)}(p, i)\left(\begin{array}{c}n+r p+\sigma-i \\ r p+\sigma\end{array}\right)$ (the right-hand side of (22)) is

$$
\frac{1}{(r p+\sigma) !} \sum_{i=0}^{r p+\sigma} A_{a, b, r}^{\left(\alpha_{s}, \beta_{s}, r_{s}, p_{s}\right)}(p, i) .
$$

The conclusion (80) comes from equating (81) and (82).

In particular, the sum of generalized Eulerian numbers $\sum_{i=0}^{r} A_{a, b, r}(1, i)$ of the first row of the corresponding generalized Eulerian numbers triangle, is equal to $a^{r}$.

One more example: the sum $\sum_{i=0}^{9} A_{1,0,3}(3, i)$ is $1+54+405+760+405+54+1=1680$ (see GENT2 in Subsection 2.1). According to (80), we have $\sum_{i=0}^{9} A_{1,0,3}(3, i)=((3)(3)) ! \frac{1}{(3 !)^{3}}=$ 1680. In fact, as Proposition 4 says, the sum $\sum_{i=0}^{9} A_{1, b, 3}(3, i)$ is equal to 1680 , for any $b \in \mathbb{C}$. For example, if $b=4$, we have the sum $\sum_{i=0}^{9} A_{1,4,3}(3, i)=64+360+880+195+306-209+$ $120-45+10-1=1680$ (see GENT4 in Subsection 2.1).

A final example: let us consider the expansion for $\left(\begin{array}{c}3 n+b \\ 2\end{array}\right)^{2}$, namely

$$
\begin{aligned}
\left(\begin{array}{c}
3 n+b \\
2
\end{array}\right)^{2}= & \left(\begin{array}{l}
b \\
2
\end{array}\right)^{2}\left(\begin{array}{c}
n+4 \\
4
\end{array}\right)+\left(-b^{4}+5 b^{3}+8 b^{2}+15 b+9\right)\left(\begin{array}{c}
n+3 \\
4
\end{array}\right) \\
& +\left(\frac{3}{2} b^{4}-12 b^{3}+\frac{3}{2} b^{2}+90 b+180\right)\left(\begin{array}{c}
n+2 \\
4
\end{array}\right) \\
& +\left(-b^{4}+11 b^{3}-28 b^{2}-63 b+261\right)\left(\begin{array}{c}
n+1 \\
4
\end{array}\right)+\left(\begin{array}{c}
b-3 \\
2
\end{array}\right)^{2}\left(\begin{array}{c}
n \\
4
\end{array}\right) .
\end{aligned}
$$

Even though the generalized Eulerian numbers $A_{3, b, 2}(2, i), i=0,1,2,3,4$, do depend on $b$, the sum $\sum_{i=0}^{4} A_{3, b, 2}(2, i)$ does not. In fact, we have (according to (80)) $\sum_{i=0}^{(2)(2)} A_{3, b, 2}(2, i)=$ $((2)(2)) ! \frac{3^{(2)(2)}}{(2 !)^{2}}=486$.

Since the sum (80) does not depend on $b$ nor $\beta_{s}$, we have in particular ((80) with $\left.a=\alpha_{s}=1\right)$

$$
\sum_{i=0}^{r p+\sigma} A_{1, b, r}^{\left(1, \beta_{s}, r_{s}, p_{s}\right)}(p, i)=\sum_{i=0}^{r p+\sigma} A_{1,0, r}^{\left(1,0, r_{s}, p_{s}\right)}(p, i)=\frac{(r p+\sigma) !}{(r !)^{p} \prod_{s=2}^{l}\left(r_{s} !\right)^{p_{s}}} .
$$

Then, we can write (80) as

$$
\sum_{i=0}^{r p+\sigma} A_{a, b, r}^{\left(\alpha_{s}, \beta_{s}, r_{s}, p_{s}\right)}(p, i)=a^{r p}\left(\prod_{s=2}^{l} \alpha_{s}^{r_{s} p_{s}}\right) \sum_{i=0}^{r p+\sigma} A_{1,0, r}^{\left(1,0, r_{s}, p_{s}\right)}(p, i) .
$$

Moreover, from (84) we see that

$$
\sum_{i=0}^{r p} A_{1,0, r}(p, i)=\frac{(r p) !}{(r !)^{p}}, \text { and } \sum_{i=0}^{r_{s} p_{s}} A_{1,0, r_{s}}\left(p_{s}, i\right)=\frac{\left(r_{s} p_{s}\right) !}{\left(r_{s} !\right)^{p_{s}}}
$$


Then, by using (84) and (86), we can write (85) as

$$
\begin{aligned}
\sum_{i=0}^{r p} A_{1,0, r}(p, i) \prod_{s=2}^{l} \sum_{i=0}^{r_{s} p_{s}} A_{1,0, r_{s}}\left(p_{s}, i\right) & =\frac{(r p) !}{(r !)^{p}} \prod_{s=2}^{l} \frac{\left(r_{s} p_{s}\right) !}{\left(r_{s} !\right)^{p_{s}}} \\
& =\frac{(r p) ! \prod_{s=2}^{l}\left(r_{s} p_{s}\right) !}{(r p+\sigma) !} \sum_{i=0}^{r p+\sigma} A_{1,0, r}^{\left(1,0, r_{s}, p_{s}\right)}(p, i) \\
& =\left(\begin{array}{c}
r p+\sigma \\
r p, r_{2} p_{2}, \ldots, r_{s} p_{s}
\end{array}\right)^{-1} \sum_{i=0}^{r p+\sigma} A_{1,0, r}^{\left(1,0, r_{s}, p_{s}\right)}(p, i),
\end{aligned}
$$

where $\left(\begin{array}{c}r p+\sigma \\ r p, r_{2} p_{2}, \ldots, r_{s} p_{s}\end{array}\right)$ is the multinomial coefficient $\frac{(r p+\sigma) !}{(r p) !\left(r_{2} p_{2}\right) ! \cdots\left(r_{l} p_{l}\right) !}$. Finally, we have from (87) that

$$
\begin{aligned}
& \sum_{i=0}^{r p+\sigma} A_{1,0, r}^{\left(1,0, r_{s}, p_{s}\right)}(p, i) \\
& =a^{r p}\left(\prod_{s=2}^{l} \alpha_{s}^{r_{s} p_{s}}\right)\left(\begin{array}{c}
r p+\sigma \\
r p, r_{2} p_{2}, \ldots, r_{s} p_{s}
\end{array}\right)\left(\sum_{i=0}^{r p} A_{1,0, r}(p, i)\right) \prod_{s=2}^{l} \sum_{i=0}^{r_{s} p_{s}} A_{1,0, r_{s}}\left(p_{s}, i\right) .
\end{aligned}
$$

Thus, in order to know the sum of generalized Eulerian numbers $\sum_{i=0}^{r p+\sigma} A_{a, b, r}^{\left(\alpha_{s}, \beta_{s}, r_{s}, p_{s}\right)}(p, i)$ involved in the expansion (22), it is sufficient to know the sums of generalized Eulerian numbers involved in the simpler expansions

$$
\left(\begin{array}{l}
n \\
r
\end{array}\right)^{p}=\sum_{i=0}^{r p} A_{1,0, r}(p, i)\left(\begin{array}{c}
n+r p-i \\
r p
\end{array}\right) \text { and }\left(\begin{array}{c}
n \\
r_{s}
\end{array}\right)^{p_{s}}=\sum_{i=0}^{r_{s} p_{s}} A_{1,0, r_{s}}\left(p_{s}, i\right)\left(\begin{array}{c}
n+r_{s} p_{s}-i \\
r_{s} p_{s}
\end{array}\right)
$$

$(s=2, \ldots, l)$, respectively. For example, let us consider the expansion (35). In order to know the sum of coefficients, that is

$$
\begin{aligned}
& 102900-1131200+5651800-16939955+33908380-45136850 \\
& +58772550-21855275+13608790-7948490+1633500 \\
= & 20667150
\end{aligned}
$$

it is sufficient to know the simpler expansion

$$
\left(\begin{array}{l}
n \\
4
\end{array}\right)^{2}=\left(\begin{array}{c}
n+4 \\
8
\end{array}\right)+16\left(\begin{array}{c}
n+3 \\
8
\end{array}\right)+36\left(\begin{array}{c}
n+2 \\
8
\end{array}\right)+16\left(\begin{array}{c}
n+1 \\
8
\end{array}\right)+\left(\begin{array}{l}
n \\
8
\end{array}\right)
$$

and use (88) to see that the required sum is $3^{8}\left(\begin{array}{c}8+2 \\ 8\end{array}\right)(1+16+36+16+1)=20667150$.

Let us consider now the case of the generalized Eulerian numbers $A_{a, 0, r}(p, i)$, with $p=2$. From formulas (85) and (86) we see that the sum of the numbers $A_{a, 0, r}(2, i), i=0,1, \ldots, 2 r$, is given by $\sum_{i=0}^{2 r} A_{a, 0, r}(2, i)=a^{2 r} \frac{(2 r) !}{(r !)^{2}}$, which is ( $a^{2 r}$ times) the well-known formula for the sum of squares of binomial coefficients (see (38)), namely $\sum_{k=0}^{r}\left(\begin{array}{c}r \\ k\end{array}\right)^{2}=\left(\begin{array}{c}2 r \\ r\end{array}\right)$. Moreover, if $p=p_{s}=2$, $s=2, \ldots, l$, formula (88) looks as

$$
\sum_{i=0}^{2 \sum_{s=1}^{l} r_{s}} A_{a, b, r_{1}}^{\left(1, \beta_{s}, r_{s}, 2\right)}(2, i)=a^{2\left(r_{1}+r_{2}+\cdots+r_{l}\right)}\left(\begin{array}{c}
2\left(r_{1}+r_{2}+\cdots+r_{l}\right) \\
2 r_{1}, 2 r_{2}, \ldots, 2 r_{l}
\end{array}\right)\left(\begin{array}{c}
2 r_{1} \\
r_{1}
\end{array}\right) \cdots\left(\begin{array}{c}
2 r_{l} \\
r_{l}
\end{array}\right) .
$$


For example, from the expansion (63) we see that the corresponding sum of coefficients is $2(1+93+546)=1280$. As formula (90) says, this sum is $2^{6}\left(\begin{array}{l}6 \\ 3\end{array}\right)=1280$.

\section{$6 \quad$ Alternating row sums}

For the standard Eulerian numbers $A_{1,0,1}(p, i)$, the known result on alternating row sums is (5). In particular, we have $\sum_{i=1}^{p}(-1)^{i} A_{1,0,1}(p, i)=0$ if $p$ is even. That is, we know that $\sum_{i=1}^{p}(-1)^{i} A_{1,0, r}(p, i)=0$ when $p$ is even and $r=1$. In the following Proposition we will show that the alternating sum $\sum_{i=1}^{p}(-1)^{i} A_{1,0, r}(p, i)$ is equal to 0 when $p$ is even, and $r$ is any positive odd number.

Proposition 5. If $p$ is even and $r$ is odd, we have

(a)

$$
\sum_{i=r}^{r p}(-1)^{i} A_{1,0, r}(p, i)=0
$$

(b)

$$
\sum_{i=0}^{r(p-1)}(-1)^{i} A_{1, r, r}(p, i)=0
$$

Proof. (a) We use (57): for $i=r, r+1, \ldots, p r$, we have $A_{1,0, r}(p, r(p+1)-i)=A_{1,0, r}(p, i)$.

Thus

$$
\begin{aligned}
\sum_{i=r}^{r p}(-1)^{i} A_{1,0, r}(p, i) & =\sum_{i=r}^{\frac{r(p+1)-1}{2}}(-1)^{i} A_{1,0, r}(p, i)+\sum_{\substack{r(p+1)+1 \\
2}}^{r p}(-1)^{i} A_{1,0, r}(p, i) \\
& =\sum_{\substack{r=r \\
r(p+1)-1}}^{\frac{r(p+1)-1}{2}}(-1)^{i} A_{1,0, r}(p, i)+\sum_{i=r}^{\frac{r(p+1)-1}{2}}(-1)^{r(p+1)-i} A_{1,0, r}(p, r(p+1)-i) \\
& =\sum_{i=r}^{2}(-1)^{i}\left(A_{1,0, r}(p, i)-A_{1,0, r}(p, r(p+1)-i)\right) \\
& =0,
\end{aligned}
$$

as desired.

(b) We have $A_{1, r, r}(p, i)=A_{1,0, r}(p, r+i)$ for $i=0,1, \ldots, r(p-1)$. Then we have that (according to (91)), for $p$ even and $r$ odd,

$0=\sum_{i=r}^{r p}(-1)^{i} A_{1,0, r}(p, i)=(-1)^{r} \sum_{i=0}^{r(p-1)}(-1)^{i} A_{1,0, r}(p, r+i)=(-1)^{r} \sum_{i=0}^{r(p-1)}(-1)^{i} A_{1, r, r}(p, i)$,

from where (92) follows.

See for example GENT2 in Subsection 2.1, where the alternating row sums is 0 for $p=2$ and $p=4$. 
Proposition 6. If $r$ is odd, we have

$$
\sum_{i=0}^{r p-\frac{r-1}{2}}(-1)^{i} A_{2,1, r}\left(p, i+\frac{r-1}{2}\right)=0
$$

in each of the following cases: (a) p even and $r \equiv 3 \bmod 4$. (b) $p$ odd and $r \equiv 1 \bmod 4$.

Proof. We will use (58) (Proposition 2): the generalized Eulerian numbers $A_{2,1, r}(p, i)$ have the symmetry $A_{2,1, r}\left(p, i+\frac{r-1}{2}\right)=A_{2,1, r}(p, r p-i)$, for $i=0, \ldots, r p-\frac{r-1}{2}$. Observe that in any of the cases (a) or (b), the number $r p-\frac{r-1}{2}$ is odd. Then

$$
\begin{aligned}
& \sum_{i=0}^{r p-\frac{r-1}{2}}(-1)^{i} A_{2,1, r}\left(p, i+\frac{r-1}{2}\right) \\
= & \sum_{i=0}^{\frac{1}{2}\left(r p-\frac{r-1}{2}-1\right)}(-1)^{i} A_{2,1, r}\left(p, i+\frac{r-1}{2}\right)+\sum_{i=\frac{1}{2}\left(r p-\frac{r-1}{2}+1\right)}^{r p-\frac{r-1}{2}}(-1)^{i} A_{2,1, r}\left(p, i+\frac{r-1}{2}\right) \\
= & \sum_{i=0}^{\frac{1}{2}\left(r p-\frac{r-1}{2}-1\right)}(-1)^{i} A_{2,1, r}\left(p, i+\frac{r-1}{2}\right)+\sum_{i=0}^{\frac{1}{2}\left(r p-\frac{r-1}{2}-1\right)}(-1)^{r p-\frac{r-1}{2}-i} A_{2,1, r}(p, r p-i) \\
= & \left(1+(-1)^{r p-\frac{r-1}{2}}\right)^{\frac{1}{2}\left(r p-\frac{r-1}{2}-1\right)}\left(\sum_{i=0}^{i}(-1)^{i} A_{2,1, r}\left(p, i+\frac{r-1}{2}\right)\right. \\
= & 0,
\end{aligned}
$$

as desired.

The expansion (63) ( $p=2$ and $r=3$ ) is an example of the case (a). The expansion $\left(\begin{array}{c}2 n+1 \\ 5\end{array}\right)=$ $\left(\begin{array}{c}n+3 \\ 5\end{array}\right)+15\left(\begin{array}{c}n+2 \\ 5\end{array}\right)+15\left(\begin{array}{c}n+1 \\ 5\end{array}\right)+\left(\begin{array}{l}n \\ 5\end{array}\right)(p=1$ and $r=5)$ is an example of the case (b).

In the general case of the expansion (24), we have the following result.

Proposition 7. The alternating sum of generalized Eulerian numbers $A_{a, b, r}(p, j), 0 \leq j \leq r p$, is given by

$$
\begin{aligned}
& \sum_{j=0}^{r p}(-1)^{j} A_{a, b, r}(p, j) \\
= & \frac{2^{r p+1}}{(r !)^{p}} \sum_{i_{r}=0}^{p} \cdots \sum_{i_{1}=0}^{p}\left(\prod_{j=1}^{r}\left(\begin{array}{c}
p \\
i_{j}
\end{array}\right)(b-j+1)^{p-i_{j}}\right) a^{\sum_{j=1}^{r} i_{j}} \frac{\left(1-2^{1+\sum_{j=1}^{r} i_{j}}\right) B_{1+\sum_{j=1}^{r} i_{j}}}{1+\sum_{j=1}^{r} i_{j}} .
\end{aligned}
$$

Proof. We expand the binomial $\left(\begin{array}{c}a n+b \\ r\end{array}\right)^{p}$ (in order to write it as a polynomial in $n$ ) to get

$$
\begin{aligned}
& \left(\begin{array}{c}
a n+b \\
r
\end{array}\right)^{p} \\
= & \frac{1}{(r !)^{p}} \sum_{i_{r}=0}^{p} \cdots \sum_{i_{1}=0}^{p}\left(\begin{array}{c}
p \\
i_{1}
\end{array}\right) \cdots\left(\begin{array}{c}
p \\
i_{r}
\end{array}\right) a^{i_{1}+\cdots+i_{r}} b^{p-i_{1}}(b-1)^{p-i_{2}} \cdots(b-r+1)^{p-i_{r}} n^{i_{1}+\cdots+i_{r}} .
\end{aligned}
$$


Then we have from (26), (95) and (17) that

$$
\begin{aligned}
& \frac{z \sum_{j=0}^{r p} A_{a, b, r}(p, j) z^{r p-j}}{(z-1)^{r p+1}} \\
= & \frac{1}{(r !)^{p}} \sum_{i_{r}=0}^{p} \cdots \sum_{i_{1}=0}^{p}\left(\begin{array}{c}
p \\
i_{1}
\end{array}\right) \cdots\left(\begin{array}{c}
p \\
i_{r}
\end{array}\right) a^{i_{1}+\cdots+i_{r}} b^{p-i_{1}}(b-1)^{p-i_{2}} \cdots(b-r+1)^{p-i_{r}} \\
& \times \frac{z \sum_{k=0}^{i_{1}+\cdots+i_{r}} A_{1,0,1}\left(i_{1}+\cdots+i_{r}, k\right) z^{i_{1}+\cdots+i_{r}-k}}{(z-1)^{i_{1}+\cdots+i_{r}+1}}
\end{aligned}
$$

from where we get

$$
\begin{aligned}
\sum_{j=0}^{r p} A_{a, b, r}(p, j) z^{r p-j}= & \frac{1}{(r !)^{p}} \sum_{i_{r}=0}^{p} \cdots \sum_{i_{1}=0}^{p}\left(\prod_{j=1}^{r}\left(\begin{array}{l}
p \\
i_{j}
\end{array}\right)(b-j+1)^{p-i_{j}}\right) a^{\sum_{j=1}^{r} i_{j}} \\
& \times \sum_{k=0}^{\sum_{j=1}^{r} i_{j}} A_{1,0,1}\left(\sum_{j=1}^{r} i_{j}, k\right) z^{-k+\sum_{j=1}^{r} i_{j}}(z-1)^{r p-\sum_{j=1}^{r} i_{j}}
\end{aligned}
$$

By setting $z=-1$ in (97) we obtain that

$$
\begin{aligned}
& \sum_{j=0}^{r p}(-1)^{j} A_{a, b, r}(p, j) \\
= & \frac{2^{r p}}{(r !)^{p}} \sum_{i_{r}=0}^{p} \cdots \sum_{i_{1}=0}^{p}\left(\prod_{j=1}^{r}\left(\begin{array}{l}
p \\
i_{j}
\end{array}\right)(b-j+1)^{p-i_{j}}\right)\left(\frac{a}{2}\right)^{\sum_{j=1}^{r} i_{j}} \sum_{k=0}^{\sum_{j=1}^{r} i_{j}}(-1)^{k} A_{1,0,1}\left(\sum_{j=1}^{r} i_{j}, k\right),
\end{aligned}
$$

and finally we use (5) to obtain from (98) the desired conclusion (94).

Let us see some particular cases of (94). In the case $r=1$ formula (94) looks as

$$
\sum_{j=0}^{p}(-1)^{j} A_{a, b, 1}(p, j)=2^{p+1} \sum_{i=0}^{p}\left(\begin{array}{l}
p \\
i
\end{array}\right) b^{p-i} a^{i} \frac{\left(1-2^{1+i}\right) B_{1+i}}{1+i}
$$

which can be written as

$$
\sum_{j=0}^{p}(-1)^{j} A_{a, b, 1}(p, j)=2^{p+1}\left(-b^{p} B_{1}+\sum_{i=0}^{\left\lfloor\frac{p}{2}\right\rfloor}\left(\begin{array}{c}
p \\
2 i+1
\end{array}\right) b^{p-2 i-1} a^{2 i+1} \frac{\left(1-2^{2+2 i}\right) B_{2+2 i}}{2+2 i}\right) .
$$

With $p=1$, formula (100) says that

$$
\sum_{j=0}^{1}(-1)^{j} A_{a, b, 1}(1, j)=4\left(-b B_{1}+a \frac{\left(1-2^{2}\right) B_{2}}{2}\right)=2 b-a .
$$

In fact, the expansion is $\left(\begin{array}{c}a n+b \\ 1\end{array}\right)=b\left(\begin{array}{c}n+1 \\ 1\end{array}\right)+(a-b)\left(\begin{array}{l}n \\ 1\end{array}\right)$, and the alternating sum of coefficients $b-(a-b)=2 b-a$. 
With $p=2$ formula (100) says that

$$
\sum_{j=0}^{2}(-1)^{j} A_{a, b, 1}(2, j)=8\left(-b^{2} B_{1}+2 b a \frac{\left(1-2^{2}\right) B_{2}}{2}\right)=4 b^{2}-4 a b .
$$

In fact, the expansion is $\left(\begin{array}{c}a n+b \\ 1\end{array}\right)^{2}=b^{2}\left(\begin{array}{c}n+2 \\ 2\end{array}\right)+\left(a^{2}+2 a b-2 b^{2}\right)\left(\begin{array}{c}n+1 \\ 2\end{array}\right)+(a-b)^{2}\left(\begin{array}{c}n \\ 2\end{array}\right)$, and the alternating sums of coefficients is $b^{2}-\left(a^{2}+2 a b-2 b^{2}\right)+(a-b)^{2}=4 b^{2}-4 a b$.

In the case $r=2$, formula (94) looks as

$$
\sum_{j=0}^{2 p}(-1)^{j} A_{a, b, 2}(p, j)=2^{p+1} \sum_{i_{2}=0}^{p} \sum_{i_{1}=0}^{p}\left(\begin{array}{c}
p \\
i_{1}
\end{array}\right)\left(\begin{array}{c}
p \\
i_{2}
\end{array}\right) a^{i_{1}+i_{2}} b^{p-i_{1}}(b-1)^{p-i_{2}} \frac{\left(1-2^{i_{1}+i_{2}+1}\right) B_{i_{1}+i_{2}+1}}{i_{1}+i_{2}+1} .
$$

If $p=1$ formula (101) says that

$$
\begin{aligned}
\sum_{j=0}^{2}(-1)^{j} A_{a, b, 2}(1, j) & =4\left(-b(b-1) B_{1}+a(2 b-1) \frac{\left(1-2^{2}\right) B_{2}}{2}\right) \\
& =a-2 b-2 a b+2 b^{2} .
\end{aligned}
$$

In fact, the expansion is $\left(\begin{array}{c}a n+b \\ 2\end{array}\right)=\left(\begin{array}{c}b \\ 2\end{array}\right)\left(\begin{array}{c}n+2 \\ 2\end{array}\right)+\left(\left(\begin{array}{c}a+b \\ 2\end{array}\right)-3\left(\begin{array}{c}b \\ 2\end{array}\right)\right)\left(\begin{array}{c}n+1 \\ 2\end{array}\right)+\left(\begin{array}{c}a-b+1 \\ 2\end{array}\right)\left(\begin{array}{l}n \\ 2\end{array}\right)$, and the alternating sum of coefficients is $\left(\begin{array}{l}b \\ 2\end{array}\right)-\left(\left(\begin{array}{c}a+b \\ 2\end{array}\right)-3\left(\begin{array}{l}b \\ 2\end{array}\right)\right)+\left(\begin{array}{c}a-b+1 \\ 2\end{array}\right)=a-2 b-2 a b+2 b^{2}$.

If $p=2$ formula (101) says that

$$
\begin{aligned}
& \sum_{j=0}^{4}(-1)^{j} A_{a, b, 2}(2, j) \\
= & 8\left(-b^{2}(b-1)^{2} B_{1}+2 a b(b-1)(2 b-1) \frac{\left(1-2^{2}\right) B_{2}}{2}+2 a^{3}(2 b-1) \frac{\left(1-2^{4}\right) B_{4}}{4}\right) \\
= & 2\left(2 b^{2}-a^{3}-2 a b-4 b^{3}+2 b^{4}+6 a b^{2}-4 a b^{3}+2 a^{3} b\right) .
\end{aligned}
$$

In particular, we have $\sum_{j=0}^{4}(-1)^{j} A_{3, b, 2}(2, j)=2\left(2 b^{4}-16 b^{3}+20 b^{2}+48 b-27\right)$. If we set $b=2$, we obtain $\sum_{j=0}^{4}(-1)^{j} A_{3,2,2}(2, j)=106$, which is the value of the alternating sum $1-95+294-95+1$ (see GENT3 in Subsection 2.1).

If $b=1$, formula (102) is

$$
\sum_{j=0}^{4}(-1)^{j} A_{a, 1,2}(2, j)=16 a^{3} \frac{\left(1-2^{4}\right) B_{4}}{4}=2 a^{3} .
$$

In fact, the expansion of $\left(\begin{array}{c}a n+1 \\ 2\end{array}\right)^{2}$ is

$$
\begin{aligned}
\left(\begin{array}{c}
a n+1 \\
2
\end{array}\right)^{2}= & \frac{1}{4} a^{2}(a+1)^{2}\left(\begin{array}{c}
n+3 \\
4
\end{array}\right)+\frac{1}{4} a^{2}\left(11 a^{2}+6 a-1\right)\left(\begin{array}{c}
n+2 \\
4
\end{array}\right) \\
& +\frac{1}{4} a^{2}\left(11 a^{2}-6 a-1\right)\left(\begin{array}{c}
n+1 \\
4
\end{array}\right)+\frac{1}{4} a^{2}(a-1)^{2}\left(\begin{array}{c}
n \\
4
\end{array}\right)
\end{aligned}
$$

and the alternating sum of coefficients is

$$
-\frac{1}{4} a^{2}(a+1)^{2}+\frac{1}{4} a^{2}\left(11 a^{2}+6 a-1\right)-\frac{1}{4} a^{2}\left(11 a^{2}-6 a-1\right)+\frac{1}{4} a^{2}(a-1)^{2}=2 a^{3} .
$$


We consider now two particular cases of the alternating sum $\sum_{j=0}^{p}(-1)^{j} A_{a, b, 1}(p, j)$.

Proposition 8. (a) If $a=2$ and $b=1$ we have

$$
\sum_{j=0}^{p}(-1)^{j} A_{2,1,1}(p, j)=2^{p} E_{p}
$$

In particular we have $\sum_{j=0}^{p}(-1)^{j} A_{2,1,1}(p, j)=0$ if $p$ is odd (see case (b) of Proposition 6). (b) If $a=b=1$

$$
\sum_{j=0}^{p}(-1)^{j} A_{1,1,1}(p, j)=\frac{2^{p+1}}{p+1}\left(2^{p+1}-1\right) B_{p+1}
$$

(This is essentially a shifted version of (5).) In particular we have $\sum_{j=0}^{p}(-1)^{j} A_{1,1,1}(p, j)=0$ if $p$ is even.

Proof. We will use that

$$
\sum_{j=0}^{p}\left(\begin{array}{c}
p+1 \\
j+1
\end{array}\right) 2^{j+1}\left(1-2^{j+1}\right) B_{j+1}=(p+1) 2^{p} E_{p}\left(\frac{1}{2}\right)=(p+1) E_{p},
$$

and

$$
\sum_{j=0}^{p}\left(\begin{array}{c}
p+1 \\
j+1
\end{array}\right)\left(1-2^{j+1}\right) B_{j+1}=\frac{p+1}{2} E_{p}(1)=\left(2^{p+1}-1\right) B_{p+1},
$$

(where $E_{p}(\cdot)$ are the Euler polynomials, and $E_{p}$ are the Euler numbers). These formulas can be obtained, for example, from (24.2.4), (24.2.5), (24.2.9), (24.2.10) and (24.4.14), of [9].

(a) According to (99) we have

$$
\sum_{j=0}^{p}(-1)^{j} A_{2,1,1}(p, j)=\frac{2^{p}}{p+1} \sum_{i=0}^{p}\left(\begin{array}{c}
p+1 \\
i+1
\end{array}\right) 2^{i+1}\left(1-2^{i+1}\right) B_{i+1} .
$$

Now use (105) to obtain (103).

(b) According to (99) we have

$$
\sum_{j=0}^{p}(-1)^{j} A_{1,1,1}(p, j)=\frac{2^{p+1}}{p+1} \sum_{j=0}^{p}\left(\begin{array}{c}
p+1 \\
j+1
\end{array}\right)\left(1-2^{j+1}\right) B_{j+1} .
$$

Now use (106) to obtain (104).

For example, with $b=1$ and $p=4$ formula (103) says that $\sum_{j=0}^{4}(-1)^{j} A_{2,1,1}(4, j)=16 E_{4}=$ 80. In fact, from (62) we see that the alternating sum of coefficients is $1-76+230-76+1=$ 80.

An interesting particular case of formula (101) is when $b=\frac{1}{2}$, considered in the following Proposition. 
Proposition 9. We have the following formula for the alternating sum of generalized Eulerian numbers $A_{a, \frac{1}{2}, 2}(p, j)$,

$$
\sum_{j=0}^{2 p}(-1)^{j} A_{a, \frac{1}{2}, 2}(p, j)=(-2)^{-p}
$$

Proof. From (101) we obtain

$$
\sum_{j=0}^{2 p}(-1)^{j} A_{a, \frac{1}{2}, 2}(p, j)=2^{-p+1} \sum_{i_{2}=0}^{p} \sum_{i_{1}=0}^{p}\left(\begin{array}{l}
p \\
i_{1}
\end{array}\right)\left(\begin{array}{c}
p \\
i_{2}
\end{array}\right)(-1)^{p-i_{2}}(2 a)^{i_{1}+i_{2}} \frac{\left(1-2^{i_{1}+i_{2}+1}\right) B_{i_{1}+i_{2}+1}}{i_{1}+i_{2}+1} .
$$

Set $i_{1}+i_{2}=j$ in the sums of the right-hand side of (108) to write

$$
\begin{aligned}
& \sum_{j=0}^{2 p}(-1)^{j} A_{a, \frac{1}{2}, 2}(p, j) \\
= & 2^{-p+1} \sum_{i_{2}=0}^{p} \sum_{j=0}^{2 p}\left(\begin{array}{c}
p \\
j-i_{2}
\end{array}\right)\left(\begin{array}{c}
p \\
i_{2}
\end{array}\right)(-1)^{p-i_{2}}(2 a)^{j} \frac{\left(1-2^{j+1}\right) B_{j+1}}{j+1} \\
= & (-2)^{-p}+2^{-p+1} \sum_{i=0}^{p}\left(\begin{array}{c}
p \\
i
\end{array}\right)(-1)^{p-i} \sum_{j=1}^{2 p}\left(\begin{array}{c}
p \\
2 j-1-i
\end{array}\right)(2 a)^{j} \frac{\left(1-2^{2 j}\right) B_{2 j}}{2 j} \\
= & (-2)^{-p}+2^{-p+1}(-1)^{p} \sum_{j=1}^{2 p}(2 a)^{j} \frac{\left(1-2^{2 j}\right) B_{2 j}}{2 j} \sum_{i=0}^{p}(-1)^{i}\left(\begin{array}{c}
p \\
i
\end{array}\right)\left(\begin{array}{c}
p \\
2 j-1-i
\end{array}\right) .
\end{aligned}
$$

Now use that

$$
\sum_{i=0}^{p}(-1)^{i}\left(\begin{array}{l}
p \\
i
\end{array}\right)\left(\begin{array}{c}
p \\
2 j-1-i
\end{array}\right)=0
$$

(identity (3.32) of Gould's book [13]) to obtain the conclusion (107).

For example, the expansion of $\left(\begin{array}{c}a n+\frac{1}{2} \\ 2\end{array}\right)^{2}$ is

$$
\begin{aligned}
\left(\begin{array}{c}
a n+\frac{1}{2} \\
2
\end{array}\right)^{2}= & \frac{1}{64}\left(\begin{array}{c}
n+4 \\
4
\end{array}\right)+\frac{4 a^{4}-2 a^{2}-1}{16}\left(\begin{array}{c}
n+3 \\
4
\end{array}\right)+\frac{88 a^{4}+4 a^{2}+3}{32}\left(\begin{array}{c}
n+2 \\
4
\end{array}\right) \\
& +\frac{44 a^{4}+2 a^{2}-1}{16}\left(\begin{array}{c}
n+1 \\
4
\end{array}\right)+\frac{16 a^{4}-8 a^{2}+1}{64}\left(\begin{array}{c}
n \\
4
\end{array}\right),
\end{aligned}
$$

and the alternating sum of coefficients is

$$
\frac{1}{64}-\frac{4 a^{4}-2 a^{2}-1}{16}+\frac{88 a^{4}+4 a^{2}+3}{32}-\frac{44 a^{4}+2 a^{2}-1}{16}+\frac{16 a^{4}-8 a^{2}+1}{64}=\frac{1}{4}=(-2)^{-2} \text {. }
$$

\section{References}

[1] Carlitz, L. (1952) Note on a Paper of Shanks, Amer. Math. Monthly, 59, 4, 239-241.

[2] Carlitz, L. (1978) A Note on $q$-Eulerian Numbers, J. Combin. Theory Ser. A, 25, 90-94. 
[3] Carlitz, L. (1959) Eulerian numbers and polynomials, Math. Mag., 32, 247-260.

[4] Carlitz, L. (1954) q-Bernoulli and Eulerian numbers, Trans. Amer. Math. Soc., 76, 332-350.

[5] Carlitz, L. (1975) A combinatorial property of $q$-Eulerian numbers, Amer. Math. Monthly, $82,51-54$.

[6] Carlitz, L. (1960) Eulerian numbers and polynomials of higher order, Duke Math. J., 27, $401-423$.

[7] Carlitz, L. (1964) Extended Bernoulli and Eulerian numbers, Duke Math. J., 31, 667-689.

[8] Carlitz, L., \& Riordan, J. (1953) Congruences for Eulerian Numbers, Duke Math. J., 20, 339-343.

[9] Dilcher, K. Bernoulli and Euler Polynomials, Digital Library of Mathematical Functions, Chapter 24. Available at: http://dlmf .nist.gov/24.

[10] Euler, L. (1755) Institutiones Calculi Differentialis, Academiae Imperialis Scientiarum Petropolitanae.

[11] Foata, D. (2010) Eulerian Polynomials: from Euler's Time to the Present, in "The Legacy of Alladi Ramakrishnan in the Mathematical Sciences", Springer, 253-273.

[12] Foata, D., \& Schützenberger, M. P. (1970) Théorie géométrique des polynômes Eulériens, Lecture Notes in Mathematics, Vol. 138, Springer.

[13] Gould, H. W. (1972) Combinatorial Identities, Morgantown, W. Va.

[14] Graf, U. (2004) Applied Laplace Transforms and z-Transforms for Scientists and Engineers: A Computational Approach using a Mathematica Package, Birkhäuser.

[15] Hsu, L. C., \& Shiue, P. J. S. (1999) On certain summation problems and generalizations of Eulerian polynomials and numbers, Discrete Math., 204, 237-247.

[16] Koutras, M. V. (1994) Eulerian Numbers Associated with Sequences of Polynomials, Fibonacci Quart., 32, 44-57.

[17] Lehmer, D. H. (1982) Generalized Eulerian Numbers, J. Combin. Theory Ser. A, 32, 195-215.

[18] Lin, Z. (2013) On some generalized q-Eulerian polynomials, DMTCS Proc. AS., 439-450.

[19] Petersen, T. K. (2015) Eulerian Numbers, Birkhäuser.

[20] Savage, C. D., \& Viswanathan, G., The $1 / k$-Eulerian Polynomials, Available online at: http://www4.ncsu.edu/ savage/PAPERS/The_1_over_k_Eulerian_ Polynomials.pdf. 
[21] Shanks, E. B. (1951) Iterated Sums of Powers of the Binomial Coefficients, Amer. Math. Monthly, 58, 404-407.

[22] Stanley, R. P. (1997) Enumerative Combinatorics, Vol. 1, Cambridge Studies in Advanced Mathematics, 49, Cambridge University Press.

[23] Vilch, R. (1987) Z Transform. Theory and Applications, D. Reidel Publishing Company.

[24] Worpitzky, J. (1883) Studien über die Bernoullischen und Eulerschen Zahlen, J. Reine Angew. Math., 94, 203-232.

[25] Xiong, T., Tsao, H. P., \& Hall, J. I. (2013) General Eulerian Numbers and Eulerian Polynomials, Journal of Mathematics, Vol. 2013, ID 629132. 